\title{
Tanztheater und filmische Ästhetik. \\ Cineastische Einflüsse und Gestaltungsweisen in den Kompositionen für die Ballets Suédois 1920-1925
}

FABIAN KOLB, MAINZ

Das Paris der Zwischenkriegsjahre war Schauplatz der Avantgarden. Es waren die Années folles, die verrückten Jahre, die eine bis dahin ungekannte Kreativkraft und Experimentierfreude in allen Bereichen der Künste freisetzten. Welche zentrale Rolle hierzu namentlich das Musikund Tanztheater als Triebkraft und Katalysator für das Zusammenspiel und die Synthese der Kunstsparten und Medien einnahm, ist bekannt. ${ }^{1}$ Doch ist das Moderne-Potenzial des Pariser Balletts dabei noch kaum hinlänglich in seinen Beziehungen zum Film und zu filmisch inspirierten Ausdrucksmöglichkeiten gewürdigt worden. ${ }^{2}$ Wie sehr sich hier nicht zuletzt die Musik in ein Wechselverhältnis mit den neuesten kinematographischen Prinzipien und Techniken begab und in vielfältiger Weise auf eine cineastische Ästhetik referenzierte, lässt sich exemplarisch an den Produktionen der Ballets Suédois ersehen; jener Truppe, die - neben den Ballets Russes ${ }^{3}-$ zu den namhaftesten Tanzkompanien der Zeit zählte und sich mit ihrer Gründung im Oktober 1920 engagiert an die Spitze einer experimentellen, provokant-revolutionären Theaterästhetik stellte. ${ }^{4}$

Mit 22 Neuproduktionen, zusätzlichen Divertissements sowie nahezu 2.800 weltweiten Aufführungen in den nicht einmal fünf Jahren ihrer Existenz erhoben die im Pariser Théâtre des Champs-Élysées beheimateten Ballets Suédois unter dem finanzkräftigen Stockholmer Kunstsammler und Mäzen Rolf de Maré ${ }^{5}$ und dem Choreographen und einstigen Solo-Tänzer der

1 Exemplarisch: Roger Nichols, The Harlequin Years. Music in Paris 1917-1929, Berkeley/Los Angeles 2002; sowie Davinia Caddy, The Ballets Russes and Beyond. Music and Dance in Belle-Époque Paris, Cambridge 2012.

2 Trotz wachsenden Interesses an den Berührungspunkten von Musik und Film konzentriert sich die Forschung nach wie vor auf Filmmusik im engeren Sinne des Soundtracks. Für die wenigen Ausnahmen, die eher auf Strukturäquivalenzen blicken, wegweisend David Bordwell, "The Musical Analogy", in: Yale French Studies 60 (1980), S. 141-156; sowie Scott D. Paulin, "Cinematic' Music: Analogies, Fallacies, and the Case of Debussy", in: Music and the Moving Image 3, H. 1 (2010), S. 1-21; und Reinhard Kapp, "Neue Musik und Film bis 1930. Interdependenzen jenseits der Anwendung", in: Mth 27 (2012), S. 197-212. Aus tanzhistorischer Perspektive: Claudia Rosiny, Tanz Film: Intermediale Beziehungen zwischen Mediengeschichte und moderner Tanzästhetik, Bielefeld 2013.

3 Auch diese arbeiteten wiederholt mit Filmreferenzen, prominent etwa in Parade (1917) und Le train bleu (1924); vgl. Lynn Garafola, "Tanz, Film und die Ballets Russes”, in: Spiegelungen. Die Ballets Russes und die Künste, hrsg. von Claudia Jeschke, Ursel Berg und Birgit Zeidler, Berlin 1997, S. 164-185.

4 Vgl. Bengt Häger, Ballets Suédois, Paris 1989; Paris Modern. The Swedish Ballet 1920-1925, hrsg. von Nancy Van Norman Baer, Seattle 1995; Pascale De Groote, Ballets Suédois, Gent 2002; Arts en mouvement. Les Ballets Suédois de Rolf de Maré, Paris 1920-1925, hrsg. von Josiane Mas, Montpellier 2008; sowie Karin Dietrich, Die Schwedischen Ballette: Getanzte Visionen im Paris der 1920er-Jahre, Frankfurt a.M. 2015.

5 Siehe Erik Näslund, Rolf de Maré - Art Collector, Ballet Director, Museum Creator, Stockholm 2009. 
schwedischen Hofoper Jean Börlin ${ }^{6}$ das Ballett zu einer spartenübergreifenden, dezidiert multimedial geprägten Gesamtkunstform. Hierzu verstanden sie es, die führenden Avantgardekünstler - visionäre Literaten, bahnbrechende Bühnen- und Kostümdesigner und originelle Komponisten - zusammenzuführen; sie gewannen für ihre spektakulären Stücke so herausragende Persönlichkeiten wie Paul Claudel, Jean Cocteau, Blaise Cendrars, Luigi Pirandello und Francis Picabia, Nils von Dardel, Georges Mouveau, Fernand Léger, Irène Lagut, Alexandre Alexeieff, Tsuguharu Foujita, Giorgio de Chirico und René Clair, Maurice Ravel, Alexander Glasunow, Viking Dahl, Cole Porter, Alfredo Casella, Erik Satie, Darius Milhaud, Georges Auric, Francis Poulenc, Germaine Tailleferre, Arthur Honegger usw.; und sie wurden damit in ihren multidisziplinären Tanzproduktionen zum Motor und Kreativlabor einer innovatorisch-zukunftsgewandten Theaterkunst im Sinne der Union des Arts.

„Les Ballets Suédois ont sauté à pieds joints par-dessus les lieux communs chorégraphiques", heißt es programmatisch in ihren Annoncen:

„Le Ballet moderne, c'est la Poésie, la Peinture, la Musique autant que la Danse: Synthèse de la Vie Intellectuelle d'aujourd'hui. [...] Pour les Ballets Suédois, le but est toujours le point de départ. [...] Ils ont l'amour du lendemain. [...] Et demain les Ballets Suédois iront encore plus en avant. ${ }^{\prime 7}$ "Les Ballets Suédois sont les seuls qui ,osent'. Les Ballets Suédois sont les seuls représentatifs de la vie contemporaine. [...] ils vont propager la révolution par un mouvement d'où les conventions sont chaque jour détruites pour y être remplacées par l'invention. Vive la vie". 8

Dass sich die Verve dieses Esprit fondateur, dieser bilderstürmerische Geist unbedingter Neuerung immer wieder auch an genuin filmischen Gestaltungspotenzialen entzündete und welchen Widerhall dies speziell in der Musik fand, soll im Folgenden an vier Beispielen schlaglichtartig erörtert werden. Dabei wird deutlich, dass die transmediale Einbeziehung einer kinematographischen Ästhetik, wie sie sich im Frankreich der 1910er- und 1920er-Jahre bekanntlich mit größter Innovationsfreude entfaltete, ${ }^{9}$ nicht nur das Vokabular des avantgardistischen Tanzes und der modernen Choreographie inspirierte, sondern sich - mal eher latent, mal mehr oder weniger explizit - insbesondere auch kompositorisch in cineastisch affizierten Konzepten und Gestaltungsweisen der Musik niederschlug. Gerade die Tatsache, dass sich viele der Komponisten (von Milhaud, Auric, Poulenc und Tailleferre über Honnegger bis zu Porter) ab den späten 1920er- und 1930er-Jahren dann immer wieder als höchst produktive Filmmusikschaffende hervortaten, ${ }^{10}$ lässt die Ballets Suédois speziell auch in dieser Hinsicht als fruchtbares

6 Siehe George Dorris, „Jean Borlin as Dancer and Choreographer”, in: Dance Chronicle 22 (1999), S. 167-188.

7 La Danse, November/Dezember 1924, [S. 23].

8 Ebd., [S. 1].

9 Man denke an den filmischen Impressionismus von Filmemachern wie Louis Delluc, Abel Gance, Germaine Dulac, Jean Epstein und Marcel L'Herbier oder an den Cinéma pur von Henri Chomette und René Clair. Zudem wurde die junge Filmkunst in Frankreich durch eine besonders engagierte theoretische Reflexion und Filmkritik in Journalen und Zeitungen begleitet. Auch trafen sich Film- und andere Künstler in cinephilen Clubs zu Austausch und Diskussion, darunter Komponisten wie Maurice Ravel und Arthur Honegger. Vgl. Richard Abel, French Film Theory and Criticism. A History/Anthology 1907-1929, Princeton 1988.

10 Zur französischen Filmmusik der Tonfilmzeit jüngst Hannah Lewis, French musical culture and the coming of sound cinema, New York 2019; zu Auric: Franziska Kollinger, Von der Bühne zum Film. Georges Aurics Musik der 1930er 
Experimentierfeld und als Ideenpool für filmisches Komponieren erscheinen. Insofern wären die Innovationen der Schwedischen Ballette nicht nur in Relation zum zeitgenössischen Tanzfilm (wie etwa Soul of the Cypress 1920, Danse Macabre 1922 und Ballet mécanique 1923/1924) zu diskutieren, ${ }^{11}$ sondern ebenso auch in ihrer Beziehung zur Frühgeschichte der (französischen) Filmmusik einzuordnen ${ }^{12}$ und zudem als mögliche Impulse und Modelle für ähnliche Tendenzen etwa bei Kurt Weill (zum Beispiel in Royal Palace 1927 und Konjunktur 1928) ${ }^{13}$ und in der sogenannten Zeitoper zu erwägen.

\section{Kamera-Ästhetik und perspektivische Brechung: Les Mariés de la Tour Eiffel (1921)}

Eine dezidierte Auseinandersetzung mit den medien- und technikgeschichtlichen Neuerungen der Zeit, die sich - gewissermaßen in einem ersten Schritt - deutlich einer filmischen Ästhetik annähert, prägt bereits die skandalumwitterte Produktion des Spectacle Les Mariés de la Tour Eiffel; ein Stück, uraufgeführt am 18. Juni 1921, das Börlin auf einen Entwurf von Jean Cocteau choreographierte und dessen absurd-surreales Szenario in lose assoziativem Zusammenhang um ein Hochzeitsfoto am Eiffelturm kreist. ${ }^{14}$ Der Eiffelturm als Symbol der modernen urbanen Lebenswelt und der neuen telegraphischen Technik und Kommunikation wird dabei zur Kulisse collageartig montierter Szenen, die sich um die Technisierung und eine dadurch verzerrte Wahrnehmung ranken. Im Zentrum steht die Kamera als Apparatur, die, offenbar aufgrund eines Defekts, immer neue befremdliche Bilder generiert, Illusionen und Chimären hervorbringt, einen Vogelstrauß gebiert, ${ }^{15}$ ein Fräulein im Badekostüm, ein Rad fahrendes Mädchen, einen

Jahre, Stuttgart 2019; zu Honegger: Leslie Sprout, "Composing Film Music in Theory and Practice. Honegger's Contributions to Les misérables and Rapt", in: JAMS 72 (2019), S. 43-113.

11 Hierzu Jürg Stenzl, „Zwei frühe Tanzfilme von Dudley Murphy (1897-1968). The Soul of the Cypress (1920) und Danse Macabre (1922)", in: ders., Musik/Film. Konstellationen zwischen Claude Debussy/Dudley Murphy und Hans Werner Henze/Alain Resnais, München 2016, S. 15-37. Rolf de Maré blickte indes skeptisch auf die Möglichkeiten des Tanzfilms: "Il me semble que de la danse à l'écran, il y a trop de distance à parcourir. Nous nous trouvons en face d'une question de rythme, de mesure, difficile à résoudre"; in: Scénario, 1. März 1921, zitiert bei Häger, Ballets Suédois, S. 54/56.

12 Beginnend bekanntlich schon 1908 mit Saint-Saëns; dazu Arne Stollberg, "Illustration oder Komposition? Camille Saint-Saëns' Musik zu L' Assassinat du Duc de Guise (1908) im Licht späterer Gattungskonventionen", in: Ton-Spuren aus der Alten Welt - Europäische Filmmusik bis 1945, hrsg. von Ivana Rentsch und Arne Stollberg, München 2013, S. 93-124.

13 Vgl. Panja Mücke, Musikalischer Film - Musikalisches Theater. Medienwechsel und szenische Collage bei Kurt Weill, Münster 2011; sowie dies., „.... jene Öffentlichkeit erreicht, die Musik hören möchte'. Musiktheater und Film in den 1920erJahren", in: Oper und Film. Geschichten einer Beziehung, hrsg. von Arne Stollberg u. a., München 2019, S. 123-137. Zu Weills Verbindung nach Frankreich vgl. Kurt Weill und Frankreich, hrsg. von Andreas Eichhorn, Münster/New York 2014. Wichtig für die Rezeption der Ballets Suédois in Deutschland scheint neben ihren Tourneen vor allem die intensive Berichterstattung in Journalen wie Der Querschnitt.

14 Siehe Jean Cocteau, "Les Mariés de la Tour Eiffel. Ballet satirique en un acte", in: Jean Cocteau. Théâtre complet, hrsg. von Michel Décaudin, Paris 2003, S. 31-54. Vgl. auch Lynette Miller Gottlieb, "Images, Technology, and Music. The Ballets Suédois and Les mariés de la Tour Eiffel", in: MQ 88 (2005), S. 523-555.

15 Statt des Vögelchens, das der Fotograf hervorrufen wollte; vgl. Jean Bernier, in: Comœdia illustré 8, H. 10 (Juli/ August 1921), S. 497-499, hier S. 498: „Auparavant l'appareil du photographe, détraqué ce matin là, a laissé fuir une autruche à la place, du petit oiseau qui va sortir'." 
kleinen Jungen, der die Festgesellschaft erschießt, einen Löwen, der einen General verspeist. Die Kamera, aus deren Perspektive alles gezeigt wird, wird zum eigentlichen Akteur des Geschehens, die Darsteller zu passiven Objekten, die von der Kamera beliebig entfernt und - so wie zum Beispiel der tote General - wieder ausgespuckt werden können. ${ }^{16}$ Nicht von ungefähr treten auch ein wohlhabender Kunstsammler (eine Reverenz an de Maré?) und ein Galerist in Erscheinung, um auf einer Metaebene über den künstlerischen (und pekuniären) Wert der Bilder zu spekulieren, ${ }^{17}$ ehe am Schluss alle Beteiligten in der Kamera entschwinden und mit ihr fortgerissen werden. Sämtliche sprachlichen Äußerungen kommen dazu von zwei Phonographen, die die Ereignisse - meist absurd entkoppelt - kommentieren; auch dies eben im Modus technischer Brechung und Verzerrung. ${ }^{18}$

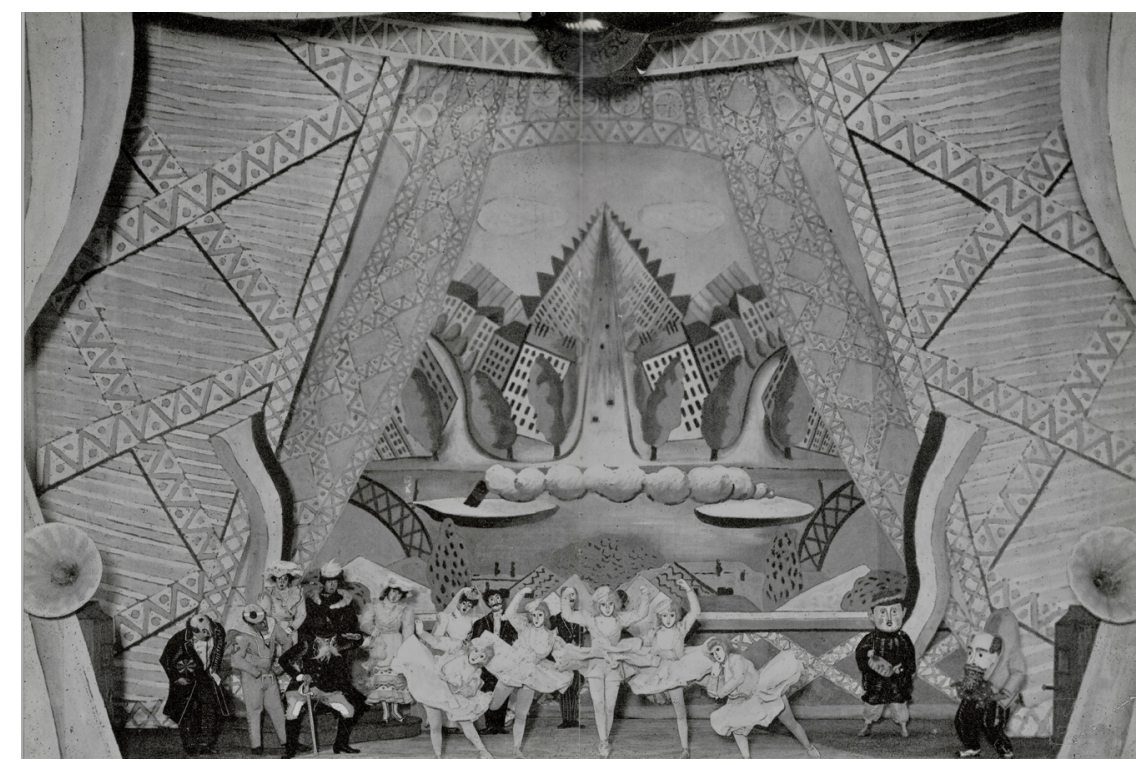

Abbildung 1.1: Les Mariés de la Tour Eiffel, Fotografie der Produktion 1921 [@ Dansmuseet Stockholm]: Bühnenprospekt mit Phonographen am linken und rechten Bühnenrand.

Hiermit konvergiert das von Irène Lagut gestaltete Bühnenbild, das - wie durch ein Objektiv - den Blick auf den Eiffelturm so ausrichtet, dass es die betrachtete Straße ist, die aufgrund der Fluchtpunktperspektive die eigentliche Gestalt des Turmes annimmt: eine doppelte Fokussierung,

16 Entsprechend die Szenenanweisung bei Cocteau, "Les Mariés de la Tour Eiffel”, S. 43: „A droite, au second plan, un appareil de photographie, de taille humaine. La chambre noire forme un corridor qui rejoint la coulisse. Le devant de l'appareil s'ouvre comme une porte, pour laisser entrer et sortir des personnages."

17 Bernier, in: Comcedia illustré, S. 498: "la noce s'ordonne pour être photographiée. Alors surviennent le riche amateur et le marchand de tableaux; celui-ci vend la noce pour un million à celui-là."

18 Dies wurde signifikanterweise als besondere Störung des ästhetischen Empfindens wahrgenommen; ebd., S. 497: „J'ai moins aimé, par contre, l'incessant dialogue des deux porte-voix qui de chaque côté de la scène commentent à pleines gueules avec une bonne humeur un peu forcée, avec une spontanéité vraiment lourde, les péripéties du ballet. L'insistance de ces voix gâte le plaisir des yeux, gâte le plaisir des oreilles, nuit gravement à ce contentement intérieur qu'on goûte devant un ballet-pantomime bien réglé." 
durch die man den Eiffelturm, gleichsam in perspektivischer Überblendung oder Mehrfachbelichtung, im Teilausschnitt und in seiner Gesamtheit zugleich wahrnimmt. Ebenso bezeichnend wie der Bühnenprospekt sind hierzu die Kostümevon Jean Hugo. Im Bemühen um möglichst klischeehafte Figuren sind sie Darstellungen im Dictionnaire Larousse entnommen ${ }^{19}$ und als puppenhafte Masken mit Pappmaschee-Körpern geformt. Im Sinne einer Commonplace-Ästhetik und Verkörperung des Gewöhnlichen dient das Outfit einer marionettenartigen Stilisierung, die sich zugleich in den stereotyp-mechanischen, immer wieder in skurrilen Posen eingefrorenen Bewegungsmustern der Tänzer niederschlug. ${ }^{20}$

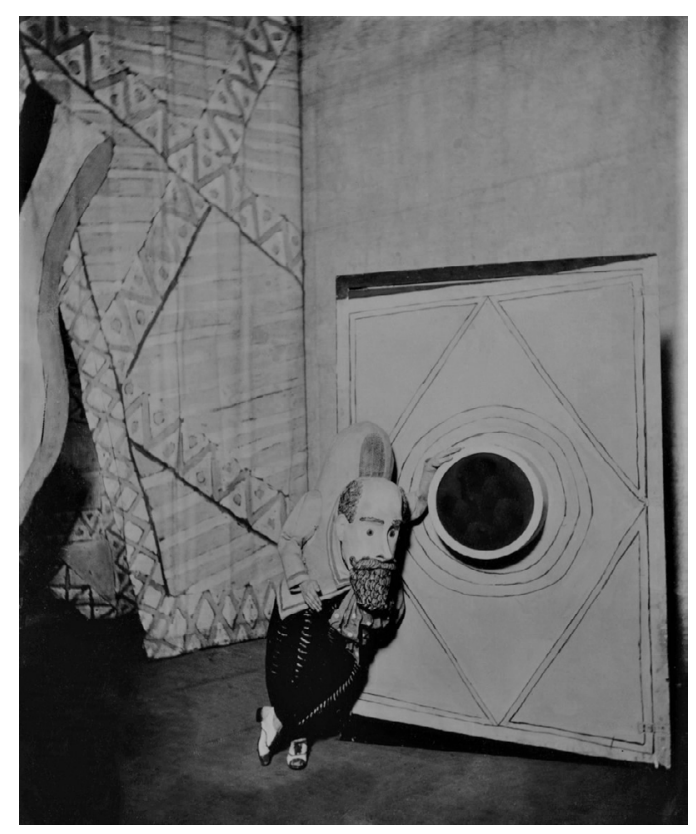

Abbildung 1.2: Les Mariés de la Tour Eiffel, Fotografie der Produktion 1921 [@ Dansmuseet Stockholm]: Axel Witzansky als Le Photographe mit seiner Kamera.

Die Musik nun fügt sich als weiteres Medium exakt in diese Art von Kamera-Ästhetik ein. Nicht nur entspricht es dem Collagen- und Montage-Charakter der Produktion, dass die Nummern im Team- bzw. als Patchwork durch verschiedene Mitglieder des Groupe de Six beigesteuert wurden. ${ }^{21}$ Sondern die einzelnen Stücke selbst folgen der Idee kurzer disparater Aperçus, gleichsam filmisch-fotografisch gefilterter Momentaufnahmen. Schon Georges Aurics "Ouverture" $\left(N^{\circ}\right.$ 1) erscheint wie ein buntes Pasticcio des Populären: Salut-Schüsse, Fanfaren, Volkstanz,

19 Siehe Jean Hugo, Le Regard de la mémoire. 1914-1945, Arles 1993, S. 188.

20 Zum Vergleich Gabriele Brandstetter, „Unter-Brechung. Inter-Medialität und Disjunktion in Bewegungs-Konzepten von Tanz und Theater der Avantgarde", in: dies., Bild-Sprung. TanzTheaterBewegung im Wechsel der Medien, Berlin 2005, S. 160-181.

21 Georges Auric: № 1 "Ouverture (Le 14 Juillet)" und № 9 "Ritournelles"; Darius Milhaud: № 2 "Marche nuptiale”, № 5 "Fugue du massacre" und № 10 "Sortie de la noce"; Francis Poulenc: № 3 "Discours du Général (Polka)" und $N^{\circ} 4$ "La Baigneuse de Trouville (Carte postale)"; Germaine Tailleferre: № 6 "Valse des Dépêches" und N ${ }^{\circ} 8$ "Quadrille"; Arthur Honegger: № 7 "Marche funèbre". 
Boulevard-Musik evozieren eine turbulente Klangkulisse passend zum Setting des Quatorze Juillet.22 Getreu der ästhetischen Maxime der "Six", Alltags- und Gebrauchsmusik zu komponieren und sich an Unterhaltungsformen wie Revue, Music Hall und Zirkus zu orientieren, ${ }^{23}$ wird hier ein Soundscape entfaltet, dessen tonmalerische Qualität die Musik in die Nähe des optischen Mediums der Kamera rückt; dies freilich ebenso im Modus technischer Verfremdung und perspektivischer Verzerrung oder Mehrfach-Fokussierung, wie er hier wesentlich durch Schichtungen und polytonale Überblendungen erzeugt wird. ${ }^{24}$

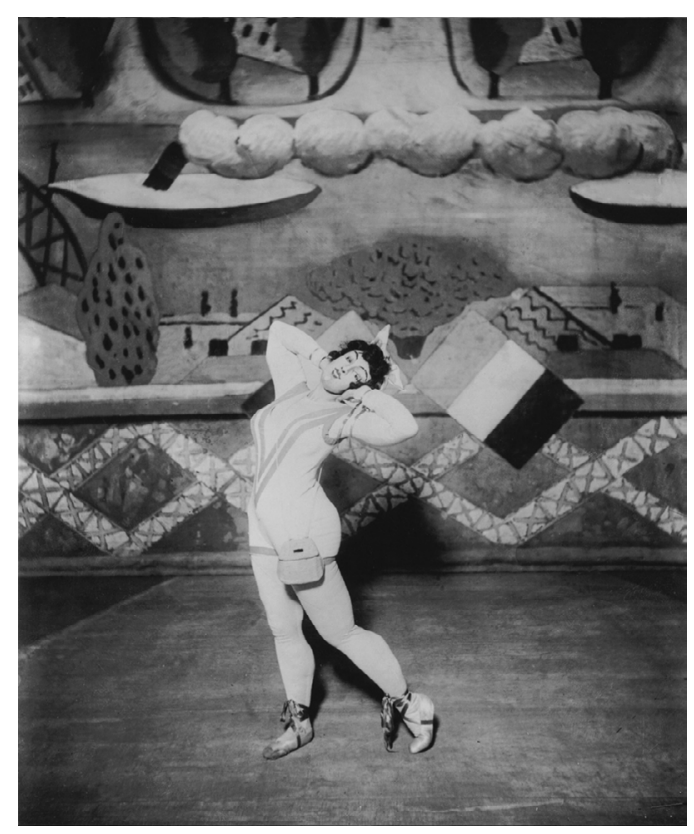

Abbildung 1.3: Les Mariés de la Tour Eiffel, Fotografie der Produktion 1921 [@ Dansmuseet Stockholm]: Carina Ari als La Baigneuse de Trouville.

Ähnliches gilt für die zirzensisch-schrille "Marche nuptiale" ( $\left.\mathrm{N}^{\circ} 2\right)$ und die grotesk-makabre „Fugue du massacre" ( $\mathrm{N}^{\circ}$ ) von Darius Milhaud; und es gilt noch einmal anders für die "Carte postale" ( $\left.\mathrm{N}^{\circ} 4\right)$ von Francis Poulenc, ${ }^{25}$ deren gezielt retrospektiv-nostalgische Fin-de-SiècleKlanglichkeit als Signum eines überkommenen Mediums (eben das der Postkarte) gedeutet

22 Vgl. auch Cocteau, "Les Mariés de la Tour Eiffel”, S. 39: „L'ouverture de Georges Auric, Le Quatorze Juillet, troupes en marche dont la musique éclate à coin d'une rue et s'éloigne, évoque aussi le charme puissant du trottoir, de la fête populaire, des estrades d'andrinople semblables à la guillotine, autour desquelles tambours et pistons font danser les dactylographes, les marins et les commis. Ces ritournelles accompagnent bas la pantomime comme l'orchestre du cirque répète un motif pendant le numéro d'acrobates. La même atmosphère circule dans la Marche nuptiale de Milhaud, le Quadrille, la Valse des dépêches, de Germaine Tailleferre, La Baigneuse de Trouville, le Discours du Général, de Poulenc."

23 Vgl. etwa Nancy Perloff, Art and the Everyday. Popular Entertainment and the Circle of Erik Satie, Oxford 1991.

24 Man beachte zugleich die ironisch-vieldeutigen Referenzen auf Stravinskys Sacre du printemps als Seitenhieb auf die Konkurrenz zu den Ballets Russes sowie inhaltlich auf die Idee des Rituellen.

25 In Parallele zur technisch korrumpierten Kommunikation via Phonographen funktioniert dagegen Poulencs "Discours du Général" ( $N^{\circ} 3$ ). Als banale Polka paraphrasiert er, so wie es sonst die Lautsprecher tun, das Geschehen und verweist in seiner augenzwinkernden Platitude auf den lapidaren Gehalt der Ansprache. 
werden mag. In seiner bewusst sentimentalischen (Über-)Pointierung gehorcht das Stück, ganz wie die kokett posierende Bade-Dame, einer Stereotypisierung. Ein klischeehaft-naiver Schnappschuss gleichsam, so wie die Telegramme in Germaine Tailleferres „DepeschenWalzer" ( $\left.\mathrm{N}^{\circ} 6\right)$ als zitathafte Montage verschiedener musikalischer Chiffren und Abbreviaturen aus dem Fundus der Ballettmusik erscheinen: überspitzte Tanztypen, überzeichnete Idiome, tanzmusikalische Topoi. Die Komposition gibt sich mit ihrem Reservoire zusammengeschnittener Ausdrucksvokabeln wie ein musikalisches Pendant zur Commonplace-Ästhetik der Kostüme. ${ }^{26}$ Das Prinzip der Überblendung schließlich kann deutlich an Arthur Honeggers „Marche funèbre" ( $\left.\mathrm{N}^{\circ} 7\right)$ beobachtet werden, die vorderhand mit ernstgemeintem Pathos daherkommt (gravitätische Punktierungen, chromatische Seufzer und absteigende Linien, dissonante Fanfaren, wehklagende Cello-Kantilene), bei der indes, sozusagen in Mehrfachbelichtung und dadurch erzeugter Uneigentlichkeit, als Gegenthema im Bassregister ironisierend der populäre Walzer aus dem zweiten Akt von Charles Gounods Faust durchscheint. ${ }^{27}$

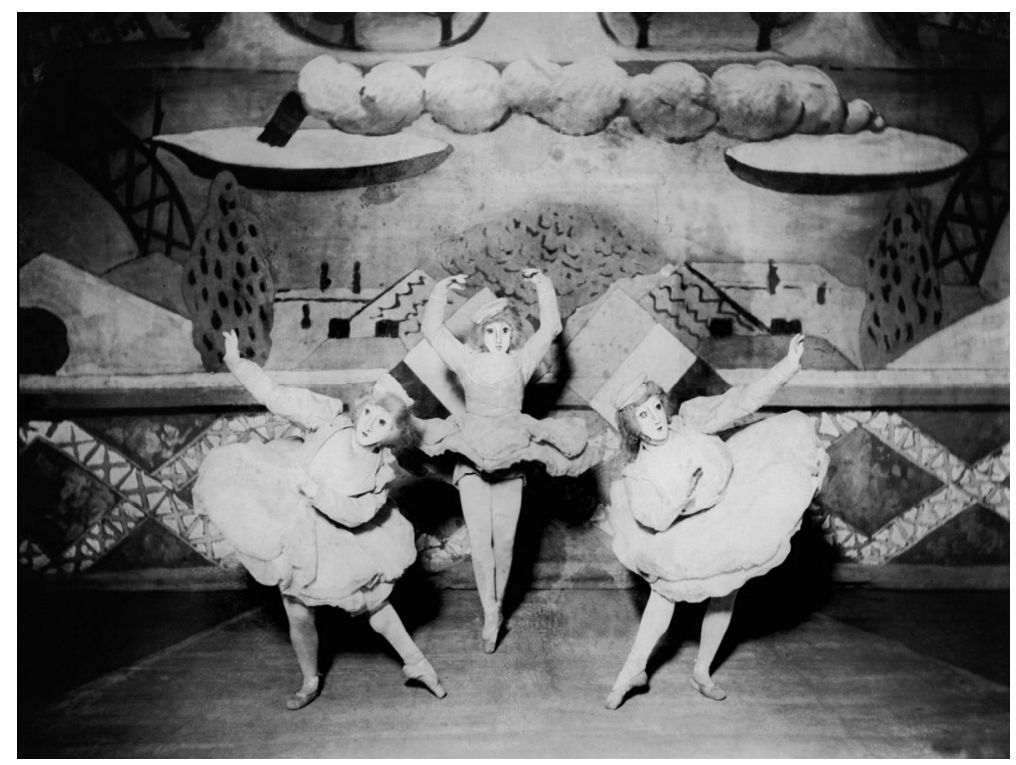

Abbildung 1.4: Les Mariés de la Tour Eiffel, Fotografie der Produktion 1921 [@ Dansmuseet Stockholm]: Torborg Stjerner, Magareta Johansson und Greta Lundberg als Dépêches.

Als bildhafte musikalische Miniaturen greifen die Stücke so jeweils in spezifischer Weise Momente und Gestaltungsideen der speziellen Kamera-Ästhetik des Balletts auf, fangen diese mit

26 Zur Ästhetik des Gemeinplatzes auch Cocteau, in: La Danse 1921, zitiert bei Häger, Ballets Suédois, S. 148: „Dans Les Mariés je montre et j'émets des lieux communs, mais je les accouple et je les présente de telle sorte qu'ils surprennent, qu'ils nous frappent avec leur jeunesse".

27 Dazu Cocteau, „Les Mariés de la Tour Eiffel”, S. 39: „Dans la Marche funèbre, Arthur Honegger s'amuse à parodier ce que nos musicographes appellent gravement: la Musique. Inutile de dire que tous tombèrent dans le panneau. À peine les premiers motifs de la marche se font-ils entendre, que les longues oreilles se dressent. Nul ne s'avisa que cette marche était belle comme un sarcasme, écrite avec un goût, un sens de l'opportunité extraordinaire. Aucun des critiques, lesquels tous s'accordent à louer ce morceau, n'y reconnut, servant de basse, la valse de Faust." 
den ihnen eigenen Möglichkeiten des Klangmediums ein. Sie reflektieren und verstärken auf musikalischer Ebene die fotografische Konzeption der Produktion. Dass sich überdies in der collageartig-heterogenen, toposhaft geprägten Reihung kurzer Nummern eine spürbare Nähe zum zeitgenössischen cineastischen Prinzip der Cue Sheet-Begleitung von Stummfilmen einstellt, ${ }^{28}$ passt hierzu, sodass sich das hier realisierte kompositorische Konzept durchaus treffend mit Milhauds Idee einer "Cinéma-Fantaisie" in Beziehung setzen ließe; eine Bezeichnung, wie sie der Komponist zeitgleich als Gattungstitel für Le bouf sur le toit ersonnen hatte. ${ }^{29}$

\section{Bildsequenzen und filmartige Collage: Within the Quota (1923)}

Ein vergleichbarer, nun in der Tat ganz unmissverständlich filmisch inspirierter Modus wurde von den Ballets Suédois gut zwei Jahre später noch einmal aufgegriffen, als am 25. Oktober 1923 der Einakter Within the Quota ${ }^{30}$ mit Musik von Cole Porter ${ }^{31}$ in der Instrumentierung von Charles Koechlin ${ }^{32}$ zur Uraufführung kam. Vom nach Frankreich übergesiedelten amerikanischen Künstlerpaar Gerald und Sara Murphy entworfen, zielt das Stück in ironisch-satirischer Weise auf die restriktive Immigrationspolitik der USA, nimmt im Titel augenzwinkernd auf die verschärften Quotenregelungen von 1917 Bezug und erzählt hierzu die Geschichte eines schwedischen Auswanderers, der mit der Hilfe einer Kinoberühmtheit und Filmproduzentin, einer offenkundigen Reverenz an Mary Pickford, seinen American Dream verwirklicht und selbst zum gefeierten Filmstar wird. Der explizite Konnex zur Filmthematik spiegelt sich dabei überaus sinnfällig in der szenischen Umsetzung der Produktion.

Auch hier ist bereits der von Murphy gestaltete Bühnenprospekt mit seiner ins Große projizierten (nota bene: projizierten!) Zeitungs-Collage aus fiktiven Schlagzeilen und Bildern der Boulevard-Presse (nach dem Vorbild der Papiers collés) repräsentativ für die alles bestimmende Zitate- und Montage-Ästhetik des Stücks, wie sie sich ebenso in den typisierten Kostümen und stereotypen Charakteren widerspiegelt: klischeehaft-prototypische Vertreter der amerikanischen Gesellschaft, der Leinwand entstiegene Protagonisten des amerikanischen Films;

28 Zu dieser Praxis im Paris der Zeit vgl. Jürg Stenzl, Musik für über 1500 Stummfilme. Das Inventar der Filmmusik im Pariser Gaumont-Palace (1911-1928) von Paul Fosse, Wien u. a. 2017. Zudem: Music and Sound in Silent Film. From the Nickelodeon to ,The Artist', hrsg. von Ruth Barton und Simon Trezise, London/New York 2019.

29 Milhaud hatte das Stück so benannt, weil er es ursprünglich zur Verwendung als Filmmusik (zu einem Stummfilm von Charlie Chaplin) konzipiert hatte. Zur Uraufführung war es indes am 21. Februar 1920 als Musik zu einer Ballett-Inszenierung von Cocteau gekommen. Gleichwohl erschien es 1921 mit dem Untertitel "CinémaFantaisie" im Druck. Bereits 1915 hatte Milhaud im Übrigen Filmmusik zu The Beloved Vagabond von Edward José beigesteuert, 1922 folgte die Musik zu Le Roi de Camargue von André Hugon.

30 Vgl. Robert Orledge, "Cole Porter's Ballet Within the Quota", in: The Yale University Library Gazette 50, H. 1 (Juli 1975), S. 19-29; Robert M. Murdock, "Gerald Murphy, Cole Porter, and the Ballets Suédois Production of Within the Quota", in: Paris Modern. The Swedish Ballet 1920-1925, hrsg. von Nancy Van Norman Baer, Seattle 1995, S. 108-117; sowie Simon Morrison, "Landed. Cole Porter's Ballet", in: A Cole Porter Companion, hrsg. von Don M. Randel, Matthew Shaftel und Susan Forscher Weiss, Urbana 2016, S. 57-69.

31 Porters Particell ist für drei, teils vier Klaviere gesetzt.

32 In weiterem Zusammenhang vgl. Antje Müller, Cinematographische Verfahrensweisen in den Orchesterwerken von Charles Koechlin, Hofheim/Taunus 2015, zur Orchestrierung von Within the Quota S. $198 \mathrm{f}$. 
cineastische Karikaturen aus dem zeitgenössischen US-Kino wie die snobistisch-glamouröse Millionärserbin à la Gloria Swanson, der prüde Puritaner und Prohibitionist, ein farbiger Gentleman aus dem Vaudeville-Theater, die verruchte Jazz-Diva und Femme fatale als Mischung aus Gilda Grey und Ann Pennington, ein Cowboy und ein Sherriff, die SweetheartType der Pseudo-Mary Pickford und ein Filmkritiker. ${ }^{33}$ Sie alle betreten in assoziativer Folge unvermittelt die Bühne, ziehen wie Filmsequenzen an dem Immigranten vorbei und posieren in mechanisch überformten Bewegungsmustern und stilisierten Attitüden ${ }^{34}$ vor einem Kameramann, der das Geschehen - gleichsam auf einer Metaebene - filmisch dokumentiert, um die Kamera am Ende sogar, die ,vierte Wand' durchbrechend, direkt ins Publikum zu richten. ${ }^{35}$

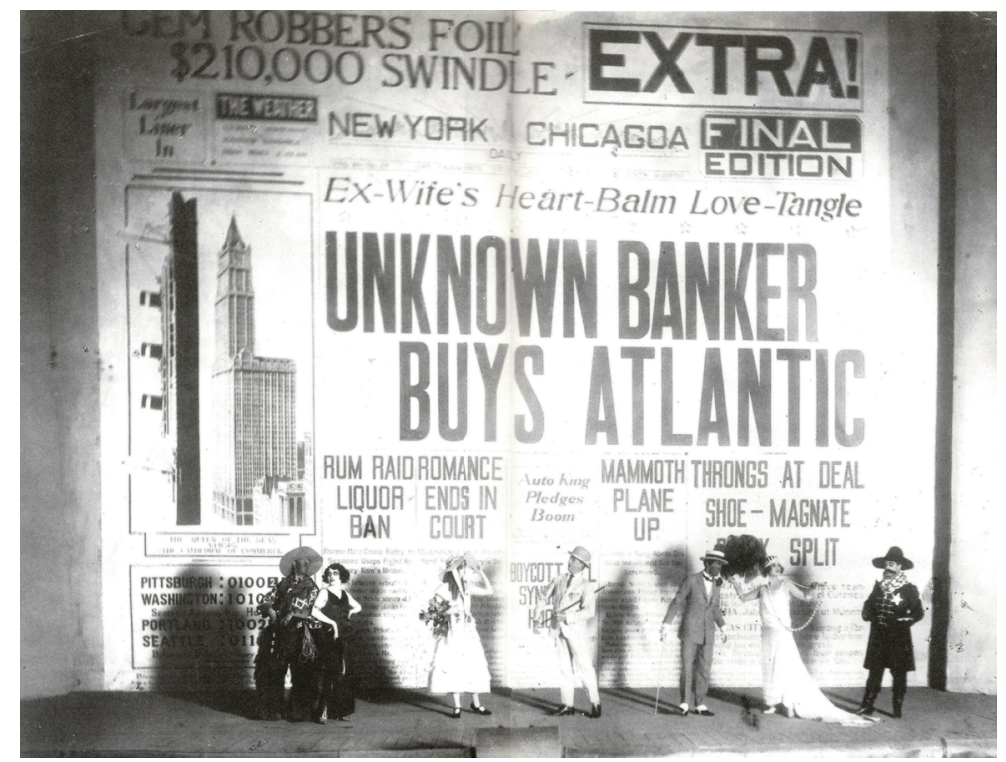

Abbildung 2.1: Within the Quota, Fotografie der Produktion 1923 [@ Dansmuseet Stockholm]: Bühnenprospekt mit Darstellern.

33 Murphy hierzu im Interview: "the object of course is to get the quintessence of Americanism out of its newspapers. [...] The characters are all out of real life. There is a Jazz Baby who is composite of Goldie Gray and Ann Pennington, a millionaires who is a study of an American woman entering the Ritz, a Sweetheart of the World who is of course Mary Pickford"; in: New York Herald, European edition, 25. Oktober 1923, S. 6.

34 Entsprechend die Kritik anlässlich der Amerika-Tournee: „Except one small bit in ,Within the Quota' there is no dancing by the members of the Swedish Ballet, but in its place is a series of poses, in which the grotesque and the ungraceful are accentuated to the highest degree"; Billboard, 8. Dezember 1923, S. 31.

35 Vgl. die Synopse der Revue de France, zitiert bei Häger, Ballets Suédois, S. 44: „Toile de fond: une immense page de journal américain, comme projetée sur un écran; elle présente des nouvelles sensationnelles bien choisies pour créer l'atmosphère américaine: Unknown banker buys Atlantic ,Un banquier inconnu achète I'Atlantique', etc. Les personnages américains viennent tour à tour dire, sans mots, par leur costume américain et leur mimique américaine: ,L'Amérique est le pays des contrastes.' Devant l'immigrant, encombré de valises, étonné, passe tout le carnaval new-yorkais: la milliardaire emperlée, que pourchasse le social reformer; le Nègre vêtu de vieux rose, avec ses ragtimes et sa bouteille de whisky, et qui est mis en fuite par l'agent du fisc; la femme déhanchée que vient moraliser un sobre quaker; le cow-boy brandissant ses revolvers; et, quand le malheureux immigrant semble renoncer à se faire une opinion sur le Nouveau Monde, la blonde étoile de cinéma, Sweetheart-of-theWorld. Finale échevelé: on ,tourne'; et quand les spectateurs, charmés par cet agréable sketch, applaudissent, I'homme à la manivelle braque son appareil vers la salle: on continue à ,tourner'. Spectacle tout à fait agréable, plein d'ironie, de gaieté, de mouvement." Desgleichen Gilbert Seldes im Paris-Journal, zitiert ebd., S. 212: „Les figures qui passent devant les yeux de l'immigrant sont les héros mythiques de la vie américaine moderne, en partie comme se les représentent la moyenne des Européens d'après le cinéma, en partie tels qu'ils sont dans la réalité." 


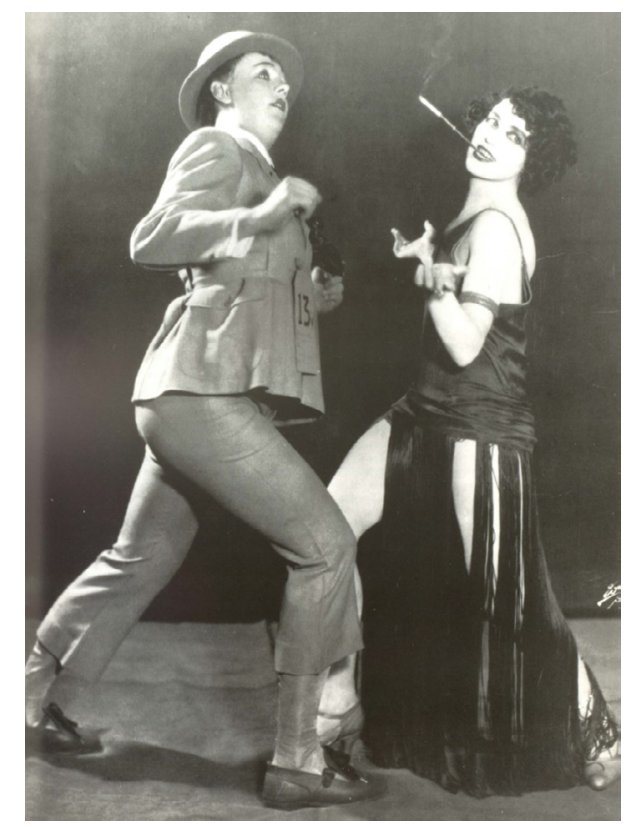

Abbildung 2.2: Within the Quota, Fotografie der Produktion 1923 [@ Dansmuseet Stockholm]: Jean Börlin als Immigrant und Ebon Strandin als Jazz Baby.

Der Verweis auf das Medium des Films wird so - analog zur medialen Präsenz der ZeitungsKulisse - zum zentralen Gegenstand des Balletts, das seine gesamte Ästhetik bestimmt. Als "Ballet-Ciné-Sketch", so der symptomatische Gattungsname, ist es letztlich eine schnitt- und montageartige Aneinanderfügung zitathafter Bildreihen: schablonenartige Momentaufnahmen des modernistischen, aber eben auch klischeegeprägten Amerika und seiner Traumfabrik. "It is nothing but a translation on to the stage of the way America looks to me from over here", bekannte Murphy. ${ }^{36}$ Entsprechend bedienen die einzelnen Auftritte auch musikalisch Images der Neuen Welt: Dampfschiffe vor Ellis Island und New Yorker Autohupen als metropolitaner Soundscape im "Opening" ( $\left.N^{\circ} 1\right)$, Foxtrott und Begleitmusik eines Kino-Pianisten bei der "Heiress" ( $\left.\mathrm{N}^{\circ} 2\right)$, Blues, Ragtime und Shimmy bei "Colored Gentleman" (No 4) und "Jazz Baby" $\left(N^{\circ} 6\right),{ }_{1}^{37}$ Schlagermelodien und Westernklänge beim "Cowboy" (N॰8), schwelgerisch-sentimentaler Hollywood-Sound als unverkennbar cineastisch inspirierte Apotheose und Happy End beim "Sweetheart Of The World" (No 9)..$^{38}$ Es sind stete akustische ,Bild'-Wechsel, Klangchiffren, musikalische Embleme und Porträts des amerikanischen Filmfiguren-Personals, überfremdet

36 New York Herald, 25. Oktober 1923, S. 6.

37 Die Uraufführung fand signifikanterweise in Kombination mit La création du monde statt, für das Milhaud ebenso Jazz-Idiome adaptierte, hier freilich nicht als Symbol des modernen Amerika, sondern einer afrikanischen Pureté primitive.

38 Die stets als "Reformer" bezeichneten Antagonisten (Prohibitionist, Sheriff etc.) werden dazwischen jeweils in einem spröde-dissonanten Idiom gezeichnet, darunter zur Charakterisierung des Puritaners aber auch Anklänge an einen Choral. 
und collageartig aneinandergefügt; es sind assoziativ-zusammenhanglose Miniaturen, KlangSequenzen, zum Teil sich überlagernd, das Ganze polytonal und polyrhythmisch verfremdet, distanziert und gefiltert wie ein medial vermittelter, auf der Kinoleinwand zu erlebender Traum, the American Dream. ${ }^{39}$

Ganz ähnlich wie bei Les Mariés de la Tour Eiffel lässt sich die kompositorische Gestaltung mit ihrer segmentierten, gezielt stereotyp ausgeprägten Folge kurzer montierter Nummern dabei auch in diesem Falle als Referenz auf das Verfahren der Cue Sheet Music und in Analogie zu Kinotheken und Filmmusikmanualen begreifen, zumal populäre Modetänze wie Ragtime, Foxtrott und Shimmy zum beliebten Standard-Arsenal von Kinomusikern zählten. Die Musik erweist sich, eingedenk der cineastischen Ausrichtung des gesamten Stücks, als filmästhetisch konzipiert.

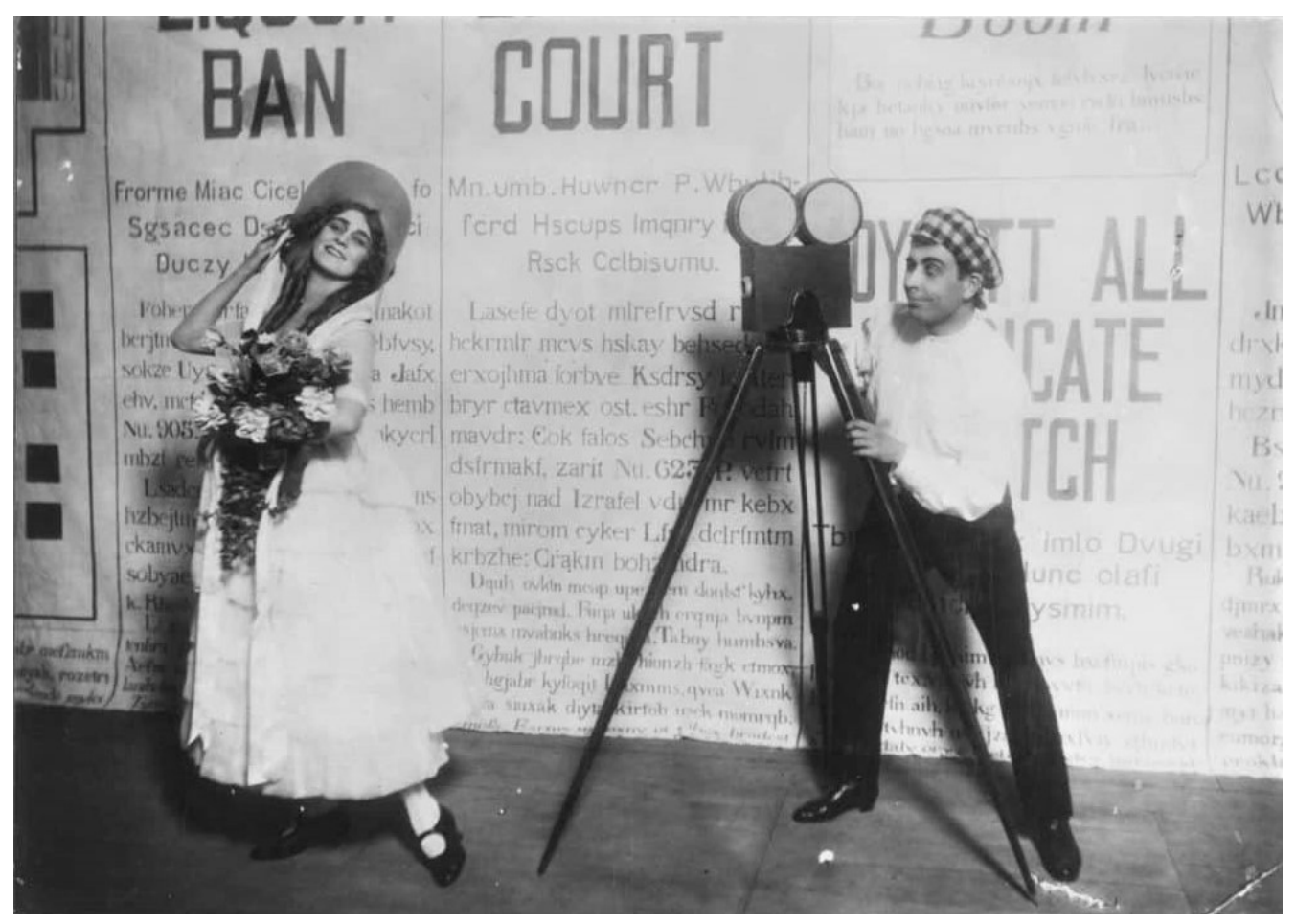

Abbildung 2.3: Within the Quota, Fotografie der Produktion 1923 [@ Dansmuseet Stockholm]: Edith von Bonsdorff als Sweetheart of the World mit Kameramann.

39 Das Traumartige wird im „Opening” exponiert, wenn in unwirklicher Klanglichkeit, gewissermaßen ,überbelichtet', das erlösende "Sweetheart"-Thema als Antizipation des Happy End eingeblendet ist, während das "Finale" wieder auf die einleitenden Takte von № 1 zurückgreift, sodass das gesamte Geschehen dazwischen gleichsam als Vision oder besser: als filmische Illusion ausgewiesen wird. Man beachte zudem die Anklänge an den Beginn von Stravinskys Petruschka, abermals eine Allusion auf die Konkurrenz-Kompanie der Ballets Russes. Hier freilich erwachen nicht Puppen eines Gauklers zum Leben, sondern Film-Protagonisten, gewissermaßen als Märchen der Moderne. 


\section{Dynamismus, Bewegung und Zeitstruktur: Skating Rink (1922)}

So wie man das Sujet von Within the Quota angesichts der lebhaften Pariser Chaplin-Begeisterung ${ }^{40}$ zugleich als Tribut an die 1917 produzierte Stummfilmkomödie The Immigrant von Charlie Chaplin auffassen kann (verquickt möglicherweise mit einer Hommage an dessen eigene Biographie), ${ }^{41}$ so hatten die Ballets Suédois der Film-Ikone bereits gut anderthalb Jahre zuvor deutliche Reverenz erwiesen, indem sie auf Chaplins Produktion The Rink aus dem Jahre 1916 rekurrierten und hierzu dezidierte Cineasten und Chaplin-Verehrer wie den Literaten und Filmkritiker Ricciotto Canudo ${ }^{42}$ und den Kubisten Fernand Léger ${ }^{43}$ gewannen. Das am 20. Januar 1922 uraufgeführte Rollschuhballett Skating Rink, ${ }^{44}$ das auf Grundlage eines 1918 verfassten „Argument" von Canudo entstand, verweist mit der Skating-Bahn und dem zum populären Breitensport gewordenen Rollschuhfahren dabei metaphorisch auf die ewige Gleichförmigkeit und Monotonie des Lebens. Allein der Künstler, der Poète, der Fou, vermag es, sich aus den permanent gleichförmig kreisenden Bahnen der Rollschuhfahrer zu lösen, und zieht damit magisch die Aufmerksamkeit einer Frau auf sich, deren Mann, als er sie zurückzugewinnen sucht ${ }^{45}$ alsbald von der Masse absorbiert und wieder in den ewigen Strudel hineingezogen wird. ${ }^{46}$

Was Canudo in seinem 21 Strophen umfassenden Gedicht durch den steten, immer wieder leicht variierten Kehrvers zum Ausdruck bringt "lls tournent, ils tournent, ils tournent le sens

40 Zur Chaplin-Rezeption Scott Douglas Paulin, On the chaplinesque in music. Studies in the cultural reception of Charlie Chaplin, Ph.D.-Diss. Princeton Univ. 2005.

41 Chaplins Einfluss auf die französische Ballettproduktion der Zeit ist in der Tat beachtlich. In diesem Zusammenhang mag daran erinnert werden, dass Chaplin 1919 in Sunnyside mit einer Traum-Tanz-Szene Nijinskys Ballets Russes-Produktion von Debussys Prélude à l'après-midi d'un faune parodiert hatte. Insgesamt wurden Chaplins pantomimische Bewegungen und Gestik vielfach mit Tanz und Ballett verglichen.

42 Canudo, der sich auch für den Kubismus engagierte, begründete 1921 den ,Club des amis du septième art' als ersten französischen Ciné-Club. Er hatte den Film als in Bewegung versetzte plastische Kunst zunächst zur sechsten Kunstform erhoben und integrierte dann auch den Tanz in sein System, wodurch der Film zur siebten Kunst wurde. Siehe Abel, French Film Theory, S. 58-66 und 291-303. Chaplin wurde von Canudo signifikanterweise als „inventeur d'un nouveau dictionnaire des gestes" bezeichnet.

43 Mehrfach schuf Léger kubistische Chaplin-Illustrationen, beginnend für Yvan Golls Die Chaplinade 1920, dann z. B. auch als Charlot-Marionette beim Ballet mécanique 1923/1924. Vgl. auch Giovanni Lista, "Léger scénographe et cinéaste", in: Fernand Léger et le spectacle, Paris 1995, S. 31-86.

$44 \mathrm{Vgl}$. auch Josiane Mas, "Skating Rink. Une musique cinétique fidèle à l'intention unanimiste et symbolique du poème de Ricciotto Canudo", in: Arthur Honegger. Werk und Rezeption, hrsg. von Peter Jost, Bern 2009, S. 275-289.

45 Hier findet sich ein expliziter Verweis aufs Kino: „L'Homme avait son couteau à la main, farouche comme dans un drame de cinéma"; zitiert nach der Erstpublikation "Skating-Ring à Tabarin. Ballet-aux-patins", in: Mercure de France CXL, 15. Mai 1920, S. 74-81, hier S. 78.

46 Siehe die bündige Zusammenfassung im Bonsoir Figaro, 21. Januar 1922: „Au milieu du cercle des patineurs enfiévrés, un homme se dresse, c'est le poète. C'est le fou. Une femme est attirée par la force qu'il dégage. Et c'est un couple merveilleux; un homme, l'amant de la femme, tente de rejoindre sa maîtresse. Mais le couple merveilleux est insaisissable. Peu à peu, malgré lui, l'homme est repris par le tournoiement général, cependant que le fou triomphant, emmène la femme". Wenngleich auf metaphorisch aufgeladener Ebene, entspricht der Plot damit durchaus der Rollschuh-Episode in The Rink: Dort ist es Chaplin, der wie der Fou aus dem Muster der Rollschuhfahrer ausbricht. Ebenso korrespondieren die Dreieckskonstellationen Chaplin - Mädchen - Rowdy / Fou - Femme - Homme. 
éperdu de toute la vie", ${ }^{47}$ das wird bei Légers Mise-en-Scène dadurch ins Bild gesetzt, dass er vor seinem abstrakten, geometrisch-kubistischen Dekor unaufhörlich Rollschuhfahrer kreisen lässt, die in ebenso kubistisch geformten, farblich plakativen Kostümen gekleidet sind. ${ }^{48}$ Dank eines gewissen Silhouetten-Effekts entsteht ein quasi-kaleidoskopartiges, in permanenter Bewegung befindliches Bühnenbild; die Produktion wird, so der Kunstkritiker Maurice Raynal, zum "jeux de formes colorées mises en mouvement" ${ }^{\prime 4}$. Getreu des von Léger formulierten Konzepts ist es ein "décor mobile", ein mechanistisch-dynamisiertes "objet-spectacle"50 - ein „tableau animé", wie es in offenkundiger Analogie zur Filmkunst hieß.51

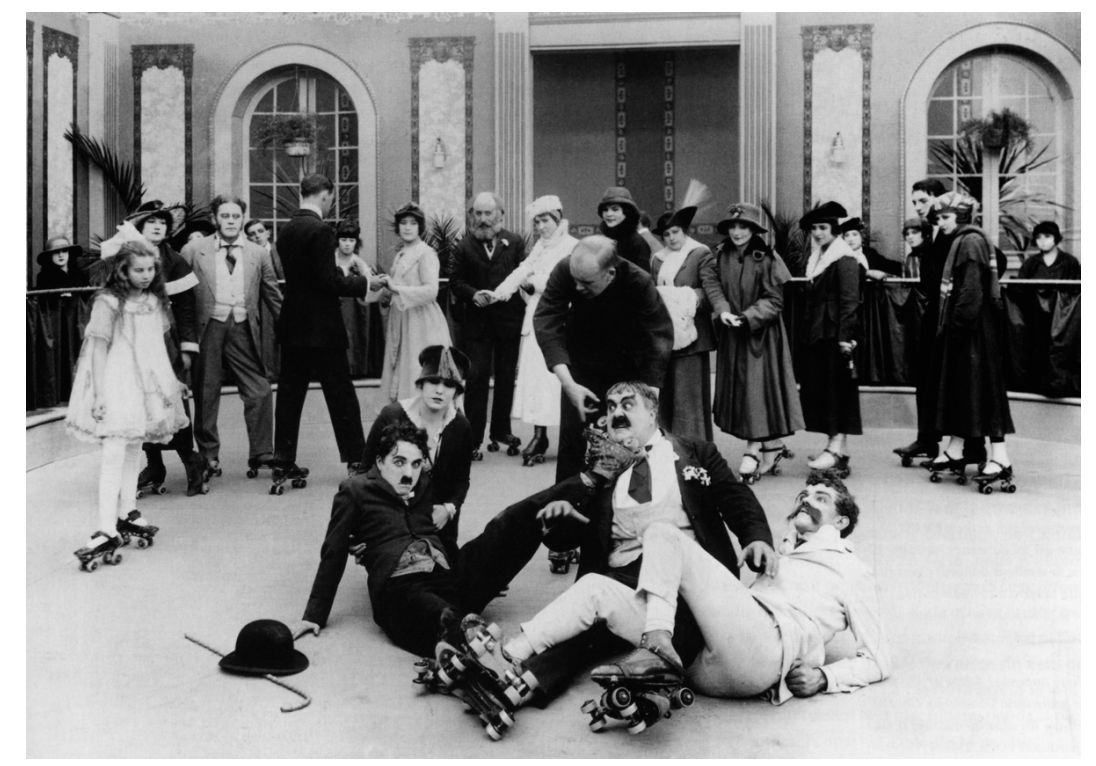

Abbildung 3.1: Charlie Chaplin, The Rink, 1916 [@ Mutual Film Corporation]: Szene auf der Rollschuhbahn.

47 Canudo, "Skating-Ring à Tabarin", S. 74-81.

48 Im kubistischen Design von Bühne und Kostümen zeigt sich eine große Nähe zum kurz darauf von Léger gestalteten Ballet mécanique. Ebenso stechen Parallelen zu Schlemmers Triadischem Ballett ins Auge; zu diesem: Thomas Betzwieser, „Zwischen Skizze und Derivat. Annäherungen an Hindemiths Musik zu Oskar Schlemmers Triadischem Ballett (1926)", in: Hindemith-Jahrbuch 37 (2008), S. 48-82. Mögliche Einflüsse der Ballets Suédois auf Schlemmer wären zu erwägen.

49 Maurice Raynal, „Skating Rink. Ballet de Fernand Léger", in: L'Esprit nouveau 17 (1922), o. S. Ebd.: „Le danseur doit faire partie intégrante du décor; il doit constituer un élément plastique qui sera fonction des éléments plastiques du décor". Zur Choreographie auch Judi Freeman, "Fernand Léger and the Ballets Suédois. The Convergence of Avant-Garde Ambitions and Collaborative Ideals", in: Paris Modern. The Swedish Ballet 1920-1925, hrsg. von Nancy Van Norman Baer, Seattle 1995, S. 86-107.

50 Fernand Léger, „Le ballet-spectacle, I'objet-spectacle” (1923), in: ders., Fonctions de la peinture, Paris 2004, S. 70: „Si on accroche solidement le matériel humain au matériel décor mobile, on a un champ d'étonnement considérable. La surface se décuple, le fond de la scène lui aussi est vivant. Tout peut bouger, ,la mesure humaine' qui, jusqu'ici, était dominante, disparait. L'homme devient un mécanisme comme le reste".

51 Raynal, "Skating Rink": „L'on dotera les ballets, quelque jour peut-être, de décors mobiles qui se dérouleront à la manière de films et présenteront un spectacle constamment renouvelé." 
Im Sinne einer Ent-Individualisierung führen die Darsteller keinerlei spezifische Gesten aus, sprechen nicht („Point de gestes. Surtout point de paroles"), formen als Masse nur mehr eine einzige anonymisiert fließende Bewegung („Tous les corps ne sont plus qu'un mouvement fluide"), sind gesichtslos ("Ils n'ont pas de visage“). ${ }^{52}$ Alle sind perpetuum-mobile-gleich in ihren zirkulären Rollmustern verfangen, ${ }^{53}$ wobei unterschiedliche Geschwindigkeiten der TänzerGruppen für wechselnde Überlagerungen der Bewegungen sorgen, ja filmische Effekte der Zeitlupe und des Zeitraffers suggerieren. ${ }^{54}$ Gerade vor dem Hintergrund der intertextuellen Anspielungen auf die Rollschuhsequenzen in Chaplins Rink resultiert hieraus ein insgesamt cineastisch konnotiertes Choreographie-Konzept mit einer deutlich kinematographisch geprägten Bewegungs-Ästhetik.

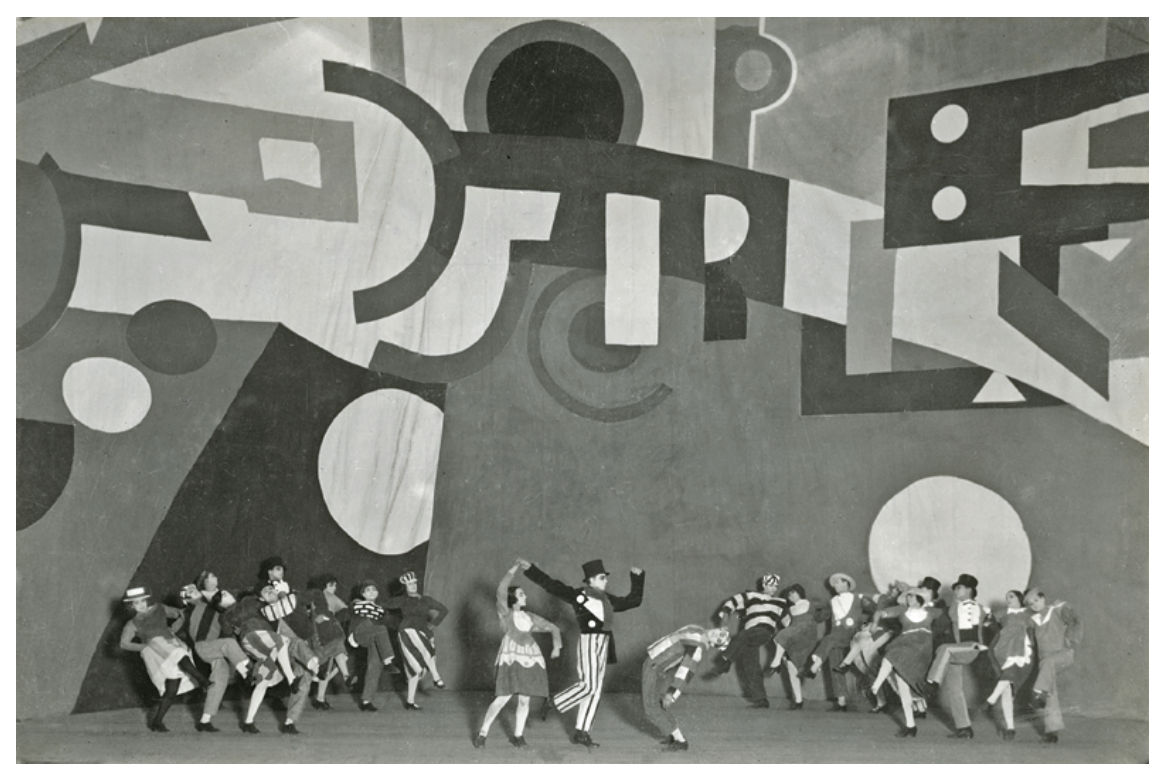

Abbildung 3.2: Skating Rink, Fotografie der Produktion 1922 [〔 Dansmuseet Stockholm]: Bühnenansicht, in der Mitte Jolanda Figoni als Femme, Jean Börlin als Fou und Kaj Smith als Homme.

Dynamismus und kinetische Qualität der Produktion betreffen dabei speziell auch die musikalische Gestaltung durch Arthur Honegger, der sich, vermittelt über Canudo, nur wenig später ebenso mit der Filmmusik zu Abel Gances La Roue (1923) befassen sollte, ${ }^{55}$ wo durchaus

52 Canudo, "Skating-Ring à Tabarin”, S. 74f.

53 Anders sind Choreographie und Bewegungskonzept in La création du monde, bei dem - ebenso von Léger in kubistischem Stil ausgestattet - die Tänzerinnen und Tänzer ganz zu mobilen Requisiten werden und kaum anderes tun, als die Masken hin und her zu bewegen. Hier wäre möglicherweise eine Nähe zur Ästhetik früher Animationsfilme (im Gefolge Émile Cohls) zu diskutieren.

54 Raynal, „Skating Rink": „Quant au décor mobile, les danseurs, il [Léger] l'ordonne par masses parallèles et contrastées, seul moyen d'éviter une monotonie que produirait l'absence de types précis. L'effet désiré sera par exemple obtenu par l'opposition de dix personnages rouges accélérés agissant contre dix personnages jaunes ralentis. Et quant aux gestes, ils affecteront une réglementation mécanique, géométrique, et d'une durée rigoureusement déterminée sous peine de délayage et de ratés."

55 Hierzu Jürg Stenzl, „Die Musik zu Abel Gances La Roue. Paul Fosse/Arthur Honegger und Robert Israel”, in: ders., Musik/Film. Konstellationen zwischen Claude Debussy/Dudley Murphy und Hans Werner Henze/Alain Resnais, 
vergleichbare Momente von Bewegung und Beschleunigung, dort der Eisenbahn, im Zentrum stehen. ${ }^{56}$ Seine Komposition zu Skating Rink evoziert dabei analog zum visuellen Eindruck des Balletts auch akustisch die Idee permanenter Rotation. 521 Takte lang konstant im 6/4-Takt „Animé", erzeugt die Musik durchgängig repetitiv mittels gleichförmiger Triller und Wechselnoten, wellenartig auf- und absteigender Skalen-Motive, Gleitbewegungen und Drehfiguren das Gefühl unaufhörlichen Kreisens in variierenden, zum Teil sich überlagernden Geschwindigkeiten. Mehr als ein Fünftel der Komposition (bis T. 114) ist ausschließlich von einem oszillierenden Perpetuum Mobile geprägt, motorisch stabil, ja monoton in Achteln mit regelmäßigen Sechzehntel-Impulsen, vor dem sich nacheinander unterschiedliche melodische Konturen abheben; allesamt Dreh-, Gleit- und Roll-Motive, die ihrerseits mal in verlangsamter, mal beschleunigter Dynamik ebenso Kreisbewegungen vollziehen, um letztlich wieder im allgemeinen Rotationsgeschehen aufzugehen; so etwa in T. 42ff. in den Celli, in T. 49ff. im Solo-Horn, in T. 65ff. abermals in den Celli, schließlich in T. 74ff. in den ersten Violinen. Das iterative, ent-individualisierte Rollen auf der Skating-Bahn wird so auch im Klangerleben nachempfunden, verdoppelt und verstärkt. Die Musik wird zur plastischen Bewegungssuggestion; und erst mit dem Auftritt des "Fou" (T. 115ff.) lagert sich - gleichsam in Nahaufnahme - in das kinetische Moment des schier unablässigen Kreisens überhaupt erstmals eine distinkte thematische Prägung in Saxophon und Englischhorn ein, die - als einzige - den ternären Duktus des 6/4-Metrums durchbricht und zwischenzeitlich eine binäre Taktordnung etabliert. ${ }^{57}$

Dass Honegger auf dieser Grundlage mit dem Hervortreten der Frau und ihrem lyrischwalzerartigen Thema (T. 173ff.) sowie des Mannes und seinen schwerfälligeren marcato-Motiven (T. 203ff.) zugleich eine großformal geschlossene Gestaltung erzielt, die durchaus einer Anlage in Sonatenform entspricht, ${ }^{58}$ korrespondiert mit seiner Bezeichnung des Stücks als "Symphonie choréographique", so wie das Moment der Formgebung - von den einzelnen motivischen Bausteinen bis hin zur Großform - mit der zirkular strukturierten Gedichtvorlage Canudos und dem kubistisch durchformten Bühnen-Konzept Légers konvergiert. Was hiervon unabhängig bleibt, ist die quasi ununterbrochen fortwirkende musikalische Rotations-Matrix in ihrer plastischen Bewegtheit und kinetischen Energie. Der Eindruck ewiger Drehbewegung als akustische Zurschaustellung dessen, was die Inszenierung mit ihrem filmisch inspirierten Décor mobile kreiert, macht die wesentliche Eigenart der Komposition aus. Die Musik sei, so bemerkten die

München 2016, S. 100-144. Honegger frequentierte Canudos Ciné-Club und wurde Gance von diesem als filmaffiner Komponist empfohlen. 1927 widmete er sich auch der Musik zu Gances Napoléon.

56 Man denke ebenso an Honeggers, Mouvement symphonique' Pacific 231 (1923).

57 Dass die Symbolik der Mensurzeichen (Kreis beim dreizeitigen Tempus perfectum, durchbrochener Kreis beim zweizeitigen Tempus imperfectum) eine Rolle für die metrische Gestaltung gespielt haben dürfte, ist nicht unwahrscheinlich.

58 Einleitung T. 1-114; Exposition T. 115-289 mit "Fou"-Thema (T. 115ff.), „Femme"-Thema (T. 173ff.) und "Homme" Thema (T. 203ff.); Durchführung T. 290-405 mit "Fou” (T. 290ff.), "Les femmes” (T. 298ff.), "Les hommes” (T. 327ff.) und "Les hommes et les femmes" (T. 372ff.); Reprise T. 415-521 mit "La danse du fou" (Schichtung von "Fou"- und „Femme"-Thema T. 415ff.). Die Themen sind in der Partitur entsprechend bezeichnet. 
Zeitgenossen, durch ein wahres Bewegungs-Fieber und einen Dreh-Rausch geprägt ( ${ }_{1} d^{\prime} u n e$ fièvre de mouvement", "d'une ivresse de glissande"), ${ }^{59}$ werde unmerklich zum bestimmenden Element des ganzen Stücks und reiße durch ihr unablässiges Kreisen alles mit sich („,mène le jeu sans qu'il y paraisse, entraînant tout dans sa grande giration jamais lasse"). ${ }^{60}$

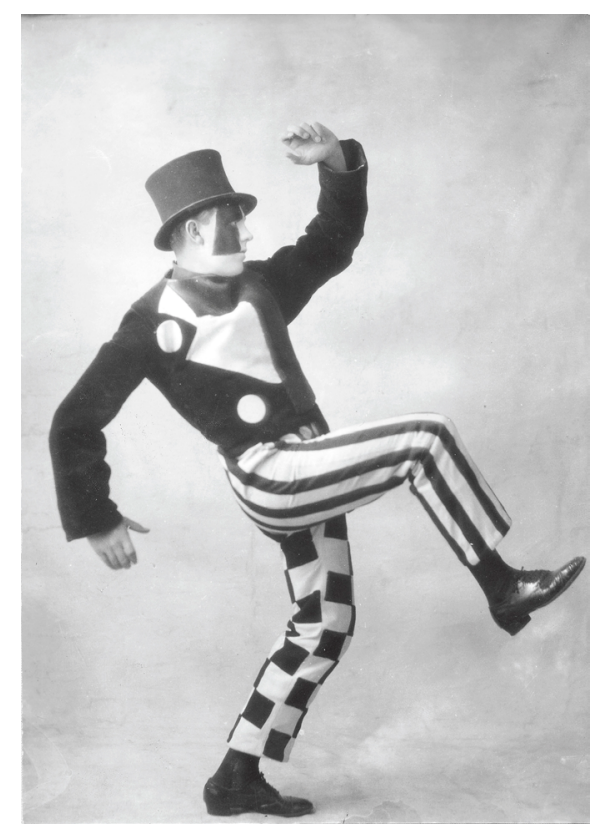

Abbildung 3.3: Skating Rink, Fotografie der Produktion 1922 [@ Dansmuseet Stockholm]: Jean Börlin als Fou.

\section{Moment und Montage: Relâche (1924)}

Noch einmal anders und zugleich am sichtlich konkretesten tritt ein filmischer Einfluss schließlich mit Blick auf Relâche hervor, ${ }^{61}$ jenes dadaistische, einer ikonoklastischen Nonsens-Ästhetik verpflichtete Ballett von Francis Picabia und Erik Satie, das provokant als "Spielpause" (frz. ,relâche') betitelt ist und das man, so die selbstironische Warnung auf den Programm-Annoncen, nur mit Sonnenbrille und Ohrstöpseln besuchen sollte: „Apportez des lunettes noires et de quoi vous boucher les oreilles". Mit seiner Uraufführung am 4. Dezember 1924 (bei der es bemerkenswerterweise mit einer Darbietung von Skating Rink gekoppelt wurde) war es die letzte realisierte Neuproduktion der Ballets Suédois ${ }^{62}$ und zugleich das erste Stück der Tanztheatergeschichte, in dem tatsächlich ein Film zum Einsatz kam. ${ }^{63}$

59 Émile Vuillermoz, in: Excelsior, 22. Januar 1922.

60 Roland-Manuel, in: L'Éclair, 23. Januar 1922.

61 Vgl. etwa Steven Moore Whiting, Satie the Bohemian. From Cabaret to Concert Hall, Oxford 1999, S. 531-557. Zu Saties Verhältnis zum Tanz insgesamt: Ornella Volta, Satie et la danse, Paris 1992 (darin zu Relâche S. 85-91).

62 Bei der letzten Aufführung von Relâche am Silvesterabend 1924 wurde als Divertissement einmalig ein CinéSketch von Picabia angefügt, bei dem simultan mehrere Tableaux vivants mit jeweils eigener Musik gezeigt wurden; Clair übernahm die Lichtregie und wechselte schnittweise die Beleuchtung der einzelnen Szenen.

631922 hatte Picabia erfolglos an Stravinsky ein Szenario für das Ballett Les Yeux chauds gesandt, das drei kurze (zweimal 15 sowie einmal 30 Sekunden dauernde) Filmsequenzen enthalten sollte: am Anfang und am Ende 
Bereits im Juli 1921 hatte Satie, damals zusammen mit dem Maler André Derain, den Plan gehegt, für die Schwedischen Ballette eine Kreation zu entwerfen, die sich vor wechselnden Filmprojektionen abspielen sollte; das Ganze eine Parodie auf das zeitgenössische Kino, bei der die Grenzen zwischen Schwarz-Weiß-Stummfilm und Liveperformance dadurch verschwimmen sollten, dass die monochrom gekleideten Tänzerinnen und Tänzer, scheinbar der Leinwand entsteigend, filmtypische Gesten persiflierten, während die Musik, gleichsam an einer intendierten Synchronizität scheiternd, entkoppelt dem Geschehen hinterherhinke. ${ }^{64}$ Doch war es zu diesem vielsagend Supercinéma ${ }^{65}$ betitelten Experiment nicht gekommen.

Nun, drei Jahre später, wurde Satie mit Relâche für ein ebenso innovatorisches medienund selbstreflexives Projekt der Ballets Suédois mit realem Filmbezug gewonnen, freilich unter etwas anderen Vorzeichen, insofern die Filmprojektionen hier separat als Vorspann und Zwischenakt in die Produktion interpoliert sind, in ihrer Dauer wie in ihrem ästhetischen Gewicht dem ,eigentlichen' Ballett indes mindestens gleichgestellt scheinen, ja letztlich zum Zentrum des Theaterabends werden. ${ }^{66}$ Das Stück insgesamt setzt dabei ganz im dadaistischen Sinne subversiv jegliche Konventionen des herkömmlichen Tanztheaters außer Kraft, wird als "Ballet instantanéiste" zu einer Art Happening. Vor blendender Scheinwerfer-Kulisse vollzieht sich ein disjunktiv-irrationales, völlig willkürlich scheinendes Aktionsgeschehen: Aus den Reihen des Publikums kommt eine Zigarette rauchende Tänzerin, die zu tanzen anfängt, sobald die Musik schweigt; man sieht einen Tänzer im Rollstuhl; ein Feuerwehrmann füllt unterdessen permanent Wasser von einem Eimer in einen anderen; Menschen entkleiden sich; provokative Plakate werden hochgehalten; die Akteure ziehen sich wieder in den Zuschauerraum zurück; eine im Auditorium sitzende Music-Hall-Sängerin wird mit einem Blumenkranz geehrt; Picabia und Satie fahren mit einem Citroën auf die Bühne.

die in rosafarbenem Licht zu realisierende Projektion der beiden Autoren, in der Mitte die eines schlüpfenden Schmetterlings; vgl. Picabias Brief vom 26. Mai 1922, in: Stravinsky. Selected Correspondence, hrsg. von Robert Craft, Bd. 2, London/Boston 1984, S. $193 f$.

64 Vgl. den Brief des Theaterdirektors Jacques Hébertot an de Maré vom 13. Juli 1921; zitiert bei Freeman, „Fernand Léger and the Ballets Suédois", S. 103, Anm. 8: „Ils [Satie et Derain] venaient tous les deux me proposer un ballet pour les Suédois: un ballet d'un nouveau genre et qui est assez amusant, ma foi. Je leur ai dit que je te soumettrais l'idée et la voici: Ce serait une parodie du Cinéma. Le décor du fond serait fait par une projection donnée sur un écran, qui remplirait tout le fond de la scène, les côtés étant d'étoffe sombre. Les personnages auraient donc l'air de sortir de l'écran tous en blanc et noir pour faire une vraie scène de cinéma. II y aurait sept ou huit scènes différentes, faciles à obtenir par le simple changement d'une projection et on se moquerait du cinéma dans l'exagération des gestes, la vitesse et par la musique qui ne serait pour ainsi dire jamais en rapport avec ce qu'on représenterait."

65 So einem Brief Saties an Derain vom 5. September 1921 zu entnehmen; Erik Satie. Correspondance presque complète, hrsg. von Ornella Volta, Paris 2000, S. 458.

66 Die beiden Ballett-Akte haben eine Dauer von ca. 20 Minuten, die Filmprojektionen sind zusammen rund 22 Minuten lang. 


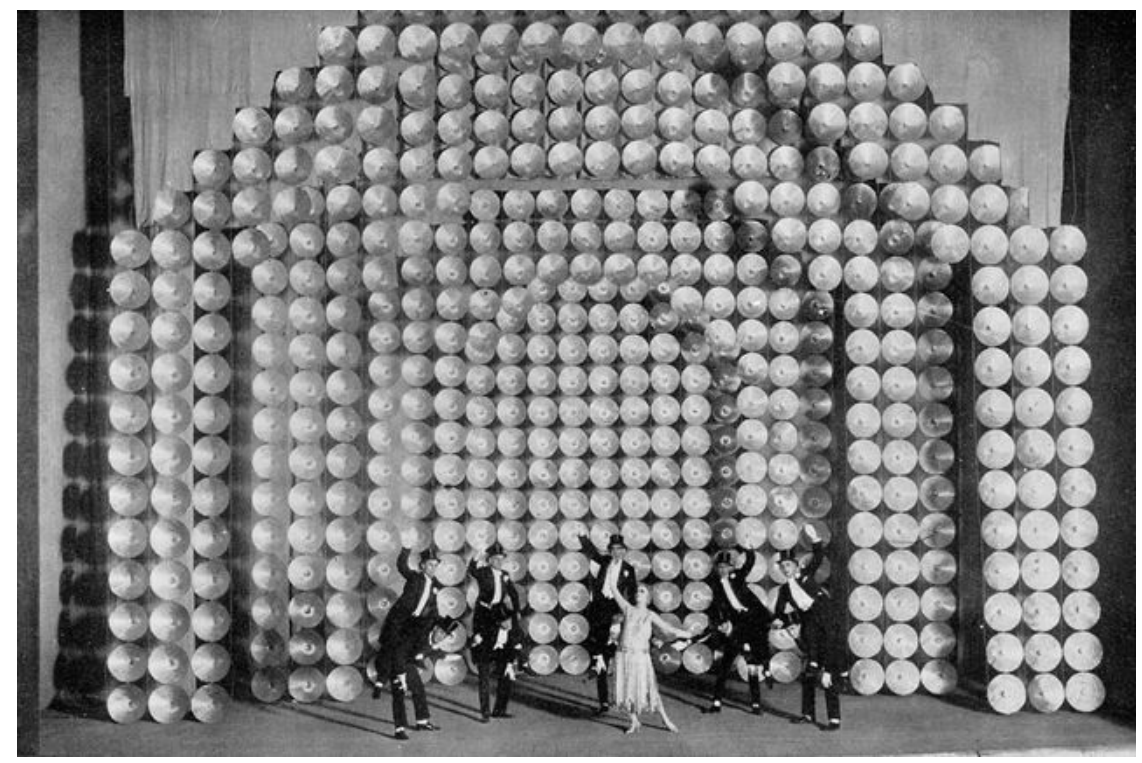

Abbildung 4.1: Relâche, Fotografie der Produktion 1924 [@ Dansmuseet Stockholm]: Bühnenansicht.

Der gänzlichen Auflösung, ja gezielt dekonstruktivistischen Negation von Bühne, Dekor und jedweder Handlungslogik ${ }^{67}$ steht dabei eine Musik zur Seite, die ebenso jegliche angestammte Kompositionsverfahren unterläuft und im Sinne von Saties Musique d'ameublement einen rein selbstzweckhaften akustischen Horizont aufspannt; musikalisches Ambiente, das sich eines Verfahrens bedient, das man in Anlehnung an Picabias Konzept des Instantaneismus, einer Augenblickskunst, als dezidiert momentbezogen charakterisieren kann. ${ }^{68}$ Die miniaturhaftaphoristischen zwei mal elf Nummern rekurrieren hierzu in absichtsvoller Banalität und Plattitüde auf formelartig zusammengefügte Elemente und kleingliedrig kompilierte Phrasen, die bewusst der Unterhaltungsmusik entlehnt sind und in ihren vielfachen Wiederholungen isoliert und entwicklungslos erscheinen. ${ }^{69}$ Es sind prägnante Bewegungsmuster und sinnfällige Idiome, musikalische Haltungen, klangliche ,Requisiten', jeweils klar umgrenzt und separiert, darunter etliche Referenzen auf bekannte Liedmelodien und Gassenhauer wie "Cadet Rousselle", "As-tu vu la cantinière", "Le Père Dupanloup" oder "J'ai du bon tabac" und "Savez-vous planter des

67 Vgl. auch Picabia in La Danse, November/Dezember 1924, [S. 18]: „Les phares d'automobiles, les colliers de perles, les formes rondes et fines des femmes, la publicité, la musique, quelques hommes en habit noir, le mouvement, le jeu, l'eau transparente et claire, le plaisir de rire, voilà Relâche [...]. Erik Satie, Borlin, Rolf de Maré, René Clair, Prieur et moi avons créé Relâche un peu comme Dieu créa la vie. Il n'y a pas de décors, il n'y a pas de costumes, il n'y a pas de nu, il n'y a qu'espace, l'espace que notre imagination aime à parcourir; Relâche est le bonheur des instants sans réflexion; pourquoi réfléchir, pourquoi avoir une convention de beauté ou de joie?"

68 Picabia hatte Relâche zur ersten Manifestation seines Instantanéisme erkoren. Die Parolen dieser Strömung finden sich auf der Titelseite der der Vorankündigung von Rélâche gewidmeten 19. und letzten Ausgabe des von Picabia herausgegebenen Dada-Journals 391 vom Oktober 1924, mündend in den Slogan: „L'Instantanéisme: ne croit qu'au mouvement perpétuel. II n'y a qu'un mouvement c'est le mouvement perpétuel!" Die Zeitung wird hier als Journal de l'Instantanéisme (pour quelque temps) betitelt.

69 Davon unberührt bleiben die strukturellen Verklammerungen der Nummern untereinander; vgl. Whiting, Satie the Bohemian, S. 552-554; sowie Robert Orledge, Satie the Composer, Cambridge 1990, S. 178-184. 
choux?". ${ }^{70}$ Der Vergleich mit Techniken filmischer Montage liegt wieder denkbar nah, so wie man - fast mehr noch als bei Les Mariés de la Tour Eiffel und Within the Quota - einen ironischen Reflex auf die Praktiken der Stummfilmbegleitung erkennen kann, auf eine mit simplen Gemeinplätzen, allbekannten Floskeln und omnipräsenten popularmusikalischen Versatzstücken operierende Kinomusik. "C'est une musique lumineuse, électrique, cinématographique, ${ }^{\text {"71 }}$ bemerkte Fernand Léger.

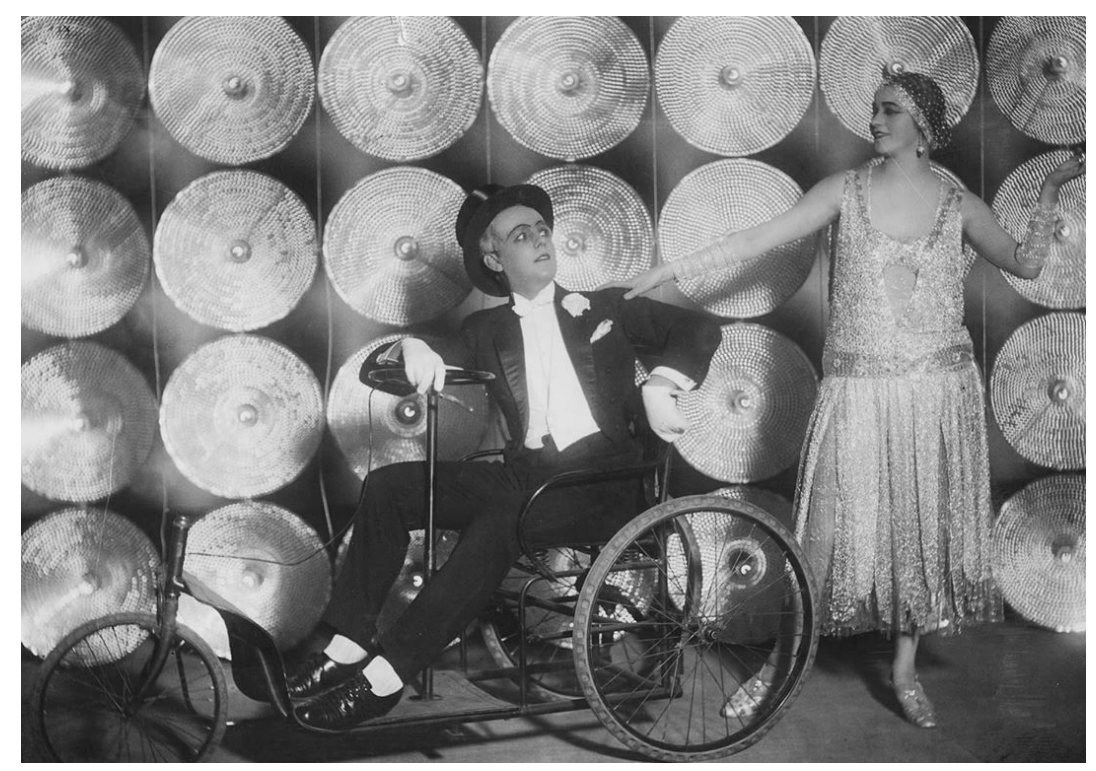

Abbildung 4.2: Relâche, Fotografie der Produktion 1924 [@ Dansmuseet Stockholm]: Jean Börlin als Un Homme und Edith von Bonsdorff als Une Femme.

Das Konzept einer cineastisch geprägten Augenblickskunst kann dabei anhand der ebenso von Satie beigesteuerten Musik zu dem Film Entr'acte von Picabia und René Clair ${ }^{72}$ deutlich demonstriert werden. ${ }^{73}$ Bereits vor dem ersten Heben des Vorhangs hatte man in einer kurzen Filmprojektion (Projectionette) Picabia und Satie vom Dach des Champs-Élysées-Theaters provokativ eine Kanone auf das Publikum abfeuern sehen, ${ }^{74}$ hiermit gewissermaßen die Leinwand durchbrechend, so wie die, vierte Wand' im Ballett selbst dann dadurch eingerissen wird, dass die Trennung zwischen Saal und Bühne aufgehoben war und die Akteure aus dem Zuschauerraum heraus das Podium betreten. ${ }^{75}$ Der Hauptfilm, der in der Pause zwischen den beiden

70 Satie bekannte: „La musique de Relâche? J'y dépeins des personnages ,en vadrouille': Pour cela, je me suis servi de thèmes populaires. Ces thèmes sont fortement ,évocateurs'... Oui: très ,évocateurs'. "Spéciaux', même"; in: La Danse, November/Dezember 1924, [S. 17].

71 Fernand Léger, "Vive Relâche”, in: Paris-Midi, 17. Dezember 1924, S. 4.

72 Clair hatte schon 1920 durch seine Mitwirkung in Loïe Fullers und Gabrielle Sorères Le Lys de la vie erste Erfahrung mit Tanz/Film-Experimenten sammeln können.

73 Vgl. auch Martin Miller Marks, Music and the Silent Film: Contexts and Case Studies, 1895-1924, Oxford/New York 1997, S. 167-185, mit Strukturübersicht auf S. 218f. Zum Film auch Karine Bouchard, "Reconstructing the Theatre Space through Cinematographic Presence: The Film Entr'acte on Stage", in: Space and Place. Exploring Critical Issues, hrsg. von Didem Kılıçkıran, Christina Alegria und Carl Haddrell, Oxford 2013, S. 43-54.

74 Hierin scheint Picabia seine Idee von 1922 (vgl. Anm. 63) aufzugreifen.

75 Ebenso sind die Darsteller in gewöhnlicher Abendgarderobe gekleidet, die Scheinwerfer der großen Lichterwand strahlen ins Publikum etc. 
Teilen des Balletts gezeigt wurde, setzt daraufhin die assoziativ freien Bildfolgen des Bühnengeschehens fort: ${ }^{76}$ Man sieht Kamin-Silhouetten, Luftballon-Puppen und Stadtansichten von Paris; ein mit Kunstbart maskierter Tänzer vollführt als Ballerina im Tutu - von unten durch eine Glasplatte gefilmt - Luftsprünge und Pirouetten; Marcel Duchamp und Man Ray spielen Schach auf dem Dach des Theaters; ein Schütze duelliert sich mit einem auf einem Wasserstrahl tanzenden Ei; ein Trauerzug zieht - erst verlangsamt, schließlich in wilder Galoppade - durch den Pariser Luna-Park, umkreist mit dem von einem Kamel gezogenen und mit Baguettekränzen behangenen Leichenwagen einen Miniatur-Eiffelturm; der Sarg gerät auf eine Achterbahn, fällt vom Wagen, und es entsteigt Börlin, der alle Trauergäste hinwegzaubert. Die am Schluss gezeigte Leinwand, auf der "Fin" verkündet wird, wird durchstoßen, indem Börlin von hinten durch sie hindurch springt - einmal mehr ein subversives Spiel mit der ,vierten Wand'. Wie die radikale Unterminierung und Demontage des herkömmlichen Balletts in den restlichen Teilen von Relâche negiert Entr'acte als Cinéma pur die narrativ-theatralen Strukturen des gewöhnlichen (kommerziellen) Handlungskinos; Tanztheater und Film assimilieren sich in ihrem dadaistischen Dekonstruktionskonzept; die Grenzen der Künste scheinen gerade durch ihre und in ihrer Negation durchbrochen, aufgelöst.

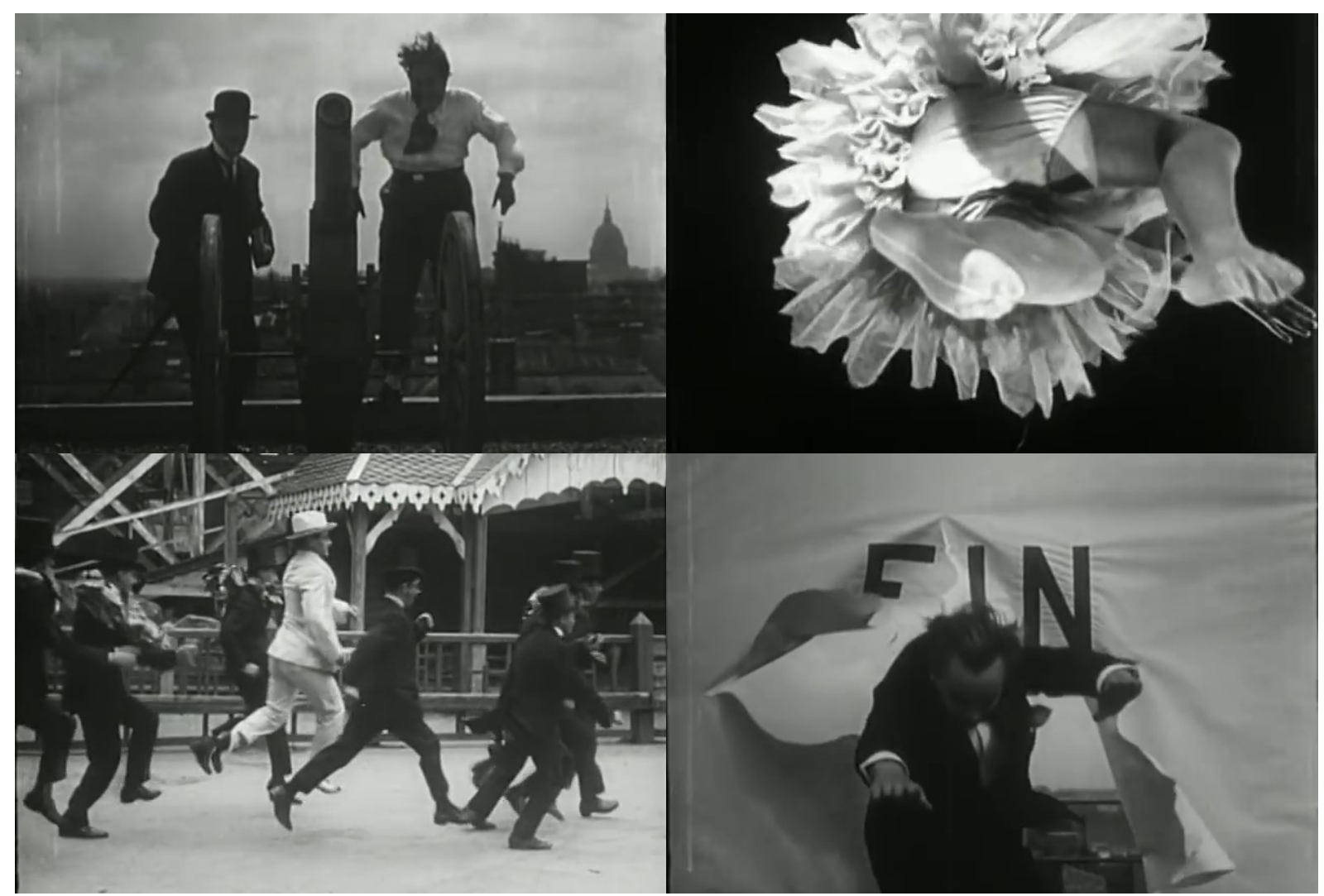

Abbildungen 4.3 - 4.6: Entr'acte, 1924: Satie und Picabia mit Kanone, Ballerina von unten, Trauerzug, "Fin”.

76 Hierzu Clair in Comœdia, 31. Oktober 1924: „Il n'y a pas une action suivie, mais une foule d'actions qui représentent la vie vue sous un angle spécial. Vous ne comprenez pas?... Ça ne fait rien. À l'écran, il ne devrait y avoir aucune convention, aucun préjugé, tout est inutile qui n'est pas vie." 
Dabei wird in Entr'acte letztlich ostentativ und in forcierter Dichte das gesamte Arsenal damaliger filmischer Gestaltungstechniken und Ausdrucksformen zur Schau gestellt: abrupte sprunghafte Schnitte, Zeitlupe und Zeitraffer, Freezing, Überblendungen, Über- und Unterperspektive, Spiegelungen, Totale und Close-up, Licht- und Schattenwirkung, Rückblenden und Antizipationen, rückwärts abgespielte Sequenzen, fotografischer Trick etc. ${ }^{77}$ Und dies läuft parallel zu den Prinzipien der Zeittaktung, des Schnitts und der Montage, wie sie auch die Musik bestimmen. In einer baukastenartigen Reihung von Formeln, Motiven und Floskeln arrangiert, oder besser: montiert Satie meist eintaktige Klangzellen bzw. Taktmuster zu größeren ostinaten Einheiten, wobei die einzelnen rhythmisch-melodischen Bausteine letztlich isoliert und verselbständigt bleiben. ${ }^{78}$ Sie stehen als Einzelheit, als Augenblick, Moment, eben instantaneistisch, wie die Einzelaufnahme eines Films; und erst durch das Prinzip der Repetition, Reihung und Addition fügen sich die Segmente schnittartig zu größeren Vier-, Acht- oder Zwölf-TaktStrecken. ${ }^{79}$ Was variiert, sind Geschwindigkeit und Ereignisdichte: mal komprimiert, mal diminuiert, mal verlangsamt, mal akzelerierend, mal in krebsgängiger Rhythmisierung. Diese Klangmuster bzw. Klangbänder werden ihrerseits zu größeren Komplexen zusammengeschnitten, wobei sie in der Fläche einer klaren Klangfelddisposition gehorchen. ${ }^{80}$ Frei von Semantisierungen ${ }^{81}$ oder gar Tonmalerei richten sich die Patterns und Sequenzen dabei in passgenauer Taktung exakt und synchron allein an der Minutage, an der Zeitstruktur der 292 Kameraeinstellungen aus, ${ }^{82}$ spielen wie diese zugleich mit reversiblen Zeitstrukturen, Antizipationen und Rückblenden. ${ }^{83}$

77 „Voici Entr'acte qui prétend donner une nouvelle valeur à l'image. [...] Dans Entr'acte, I'image ,détournée de son devoir de signifier' naît, à une existence concrète'. Rien ne me semble plus respectueux de l'avenir du film que ces balbutiements visuels dont il [Picabia] a réglé I'harmonie"; Clair in: La Danse, November/Dezember 1924, [S. 20].

78 So setzen sich die ersten 44 Takte wie folgt zusammen: $a(1+1+1+1+1+1+1+1) b(2+2) c(1+1+1+1+$ $1+1+1+1) d(1+1+1+1+1+1+1+1) e(1+1+1+1+1+1+1+1) a(1+1+1+1+1+1+1+1)$

79 Es lassen sich 58 (allermeist vier- und achttaktige) Motiveinheiten ausmachen, die zu zehn größeren Sektionen gruppiert sind und stets mit einem Wechsel der Instrumentierung einhergehen. Die Sektionen sind durch Kadenzen oder Zäsuren separiert, nur die ersten beiden fügen sich direkt aneinander; sie sind in der Partitur betitelt: [1] "Cheminées, ballons qui explosent"; [2] „Gants de boxe et allumettes"; [3] „Prises d'air, jeux d'échecs et bateaux sur les toits"; [4] "La danseuse et figures dans l'eau“; [5] „Chasseur, et début de l'enterrement”; [6] "Marche funèbre"; [7] „Cortège au ralenti"; [8] „La poursuite"; [9] „Chûte du cerceuil et sortie de Borlin"; [10] „Final (écran crevé et fin)".

80 Im Kompositionsplan hatte Satie den Sektionen bereits konkrete Tonarten zugewiesen: [1] (A F C A); [2] (A F D A); [3] (D F D); [4] (E e C E); [5] (A C F A); [6] (a d E fis [A]); [7] (A C c G e A F [C]); [8] ([h] A fis F C As B A); [9] (E G cis); [10] (A [E] A).

81 Einzige Ausnahme ist der Rückgriff auf Chopins Marche funèbre aus dessen 2. Klaviersonate b-Moll op. 35 (Satz III) in Einheit 28 zu Beginn der Leichenwagen-Szenen.

82 "Quant à Satie, le vieux maître de la jeune musique, il minutait chaque séquence avec un soin méticuleux et préparait ainsi la première composition musicale écrite pour le cinéma ,image par image' en un temps où le film était encore muet"; Clair in: L'Avant-Scène 86 (November 1968), S. $5 f$.

83 So wird das erste Pattern als Einheit 6, 14, 24, 27, 32, 37 und 55 wiederaufgegriffen. 
Satie experimentierte wie Clair mit der Verkettung von Augenblicken, mit unterschiedlichen Rhythmen und Zeittaktungen, Verlangsamungen und Beschleunigungen, mit einer Codierung von Bewegungswerten. Die Praktiken und Strukturen moderner filmischer Medientechnik scheinen so in frappierender Kongruenz von der Musik adaptiert. Nicht zuletzt hierin fand die so vielfältig intensive Auseinandersetzung der Ballets Suédois mit den neuen kinematographischen Gestaltungspotenzialen und einer cineastischen Ästhetik zu einem beachtlichen Höhepunkt, kurz bevor sich das Ensemble im März 1925 wieder auflöste. ${ }^{84}$

Zitation: Fabian Kolb, "Tanztheater und filmische Ästhetik. Cineastische Einflüsse und Gestaltungsweisen in den Kompositionen für die Ballets Suédois 1920-1925", in: Freie Beiträge zur Jahrestagung der Gesellschaft für Musikforschung 2019, hrsg. von Nina Jaeschke und Rebecca Grotjahn (= Musikwissenschaft: Aktuelle Perspektiven. Bericht über die Jahrestagung der Gesellschaft für Musikforschung 2019 in Paderborn und Detmold, Bd. 1), Detmold 2020, S. $168-190$, DOI: $10.25366 / 2020.60$.

84 Nicht (mehr) kam es zur Produktion von Cronkhite's Clocks, die auch in Hinblick auf die Verschränkung von Film und Liveperformance so etwas wie ein ultimativer Fluchtpunkt für die Avantgardekunst der Ballets Suédois hätte werden können. Edmund Wilson, der während der Amerika-Tournee 1923/1924 als Presseagent für die Kompanie tätig gewesen war, bündelte in seinem Szenario die verschiedensten Moderne-Strömungen, die man mit den Ballets Suédois in Verbindung brachte. Die Musik, für die Leo Ornstein vorgesehen war, sollte futuristisch-bruitistische Elemente (unter Beteiligung von Schreibmaschinen, Radio, Phonograph, Nietpistole, Elektromagnet, Wecker und Telefonen) sowie Jazz kombinieren; die Hauptfiguren sollten von Charlie Chaplin und einem "Negro comedian" verkörpert werden; die pantomimisch vom schwarz-weiß gekleideten Personal vor einem monochromen Hintergrund dargestellte Handlung sollte durch die Projektion von Übertiteln Erläuterung finden; und im Zentrum des Stücks sollte als längere Traumsequenz ein Film stehen: „I have written a great super-ballet of New York for the Swedish Ballet - a pantomime explained by movie captions and with a section of movie film in the middle, for which Ornstein is composing the music and in which we hope to get Chaplin to act. It is positively the most titanic thing of the kind ever projected and will make the productions of Milhaud and Cocteau sound like folk-song recitals. It is written for Chaplin, a Negro comedian, and seventeen other characters, full orchestra, movie machine, typewriters, radio, phonograph, riveter, electromagnet, alarm clocks, telephone bells, and jazz band. They may send me out to the coast in a few days to try to persuade Chaplin to take part in it. If it comes off - though it will probably start in America - they will later take it to Paris and I might go over with them"; Wilson and John Peale Bishop, 15. Januar 1924, in: Edmund Wilson. Letters on Literature and Politics, 1912-1972, hrsg. von Elena Wilson, New York 1977, S. 117. Das Libretto wurde publiziert: Edmund Wilson, Cronkhite's Clocks, in: ders., Discordant Encounters: Plays and Dialogues, New York 1926, S. 129-156. 


\section{Abstract}

The central role that avant-garde music and dance theatre played in the interplay and synthesis of the arts and media in the 1920s, particularly in Paris, is well known. However, the creative potential of ballet has hardly been recognized in its manifold relationships with film and cinematic-inspired expression. The extent to which especially ballet music interacted with the latest cinematographic principles and techniques and referred to cinematic aesthetics in a variety of ways can instructively be seen regarding the productions of the Ballets Suédois. This is discussed in this article with an exemplary look at Les Mariés de la Tour Eiffel (1921), Within the Quota (1923), Skating Rink (1922) and Relâche (1924). By that it becomes clear that the transmedia inclusion of cinematographic ideas not only inspired the vocabulary of avant-garde dance and modern choreography, but was also distinctively reflected in the conception and composition of film-affected music.

\section{Kurzvita}

Fabian Kolb ist wissenschaftlicher Mitarbeiter an der Abteilung Musikwissenschaft der Johannes Gutenberg-Universität Mainz. Nach einem Studium in Bonn und Köln promovierte er 2010 mit einer Dissertation zur französischen Symphonik um 1900. 2018 erfolgte seine Habilitation in Mainz mit einer Studie zur Instrumentalmusikkultur zwischen Spätmittelalter und Früher Neuzeit. Kolb publizierte u. a. zur Musikgeschichte des Mittelalters und der Renaissance sowie zu Musiktheater, Instrumentalmusik und musikalischer Institutionengeschichte des 18. bis 20. Jahrhunderts. 


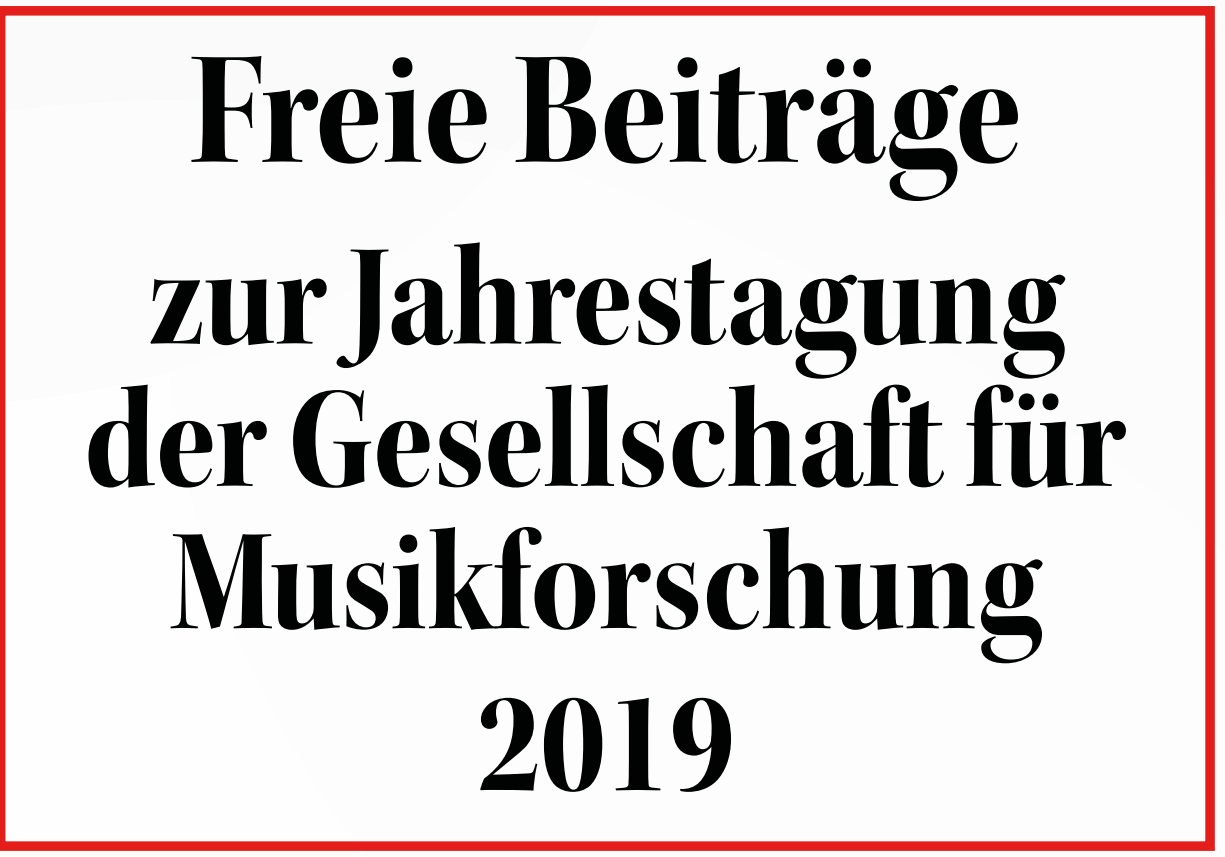

Herausgegeben von Nina Jaeschke und Rebecca Grotjahn

Musikwissenschaft: Aktuelle Perspektiven 1 
Freie Beiträge 


\section{Musikwissenschaft: Aktuelle Perspektiven}

Bericht über die Jahrestagung der Gesellschaft für Musikforschung 2019 in Paderborn und Detmold

Herausgegeben von Rebecca Grotjahn und Nina Jaeschke

Band 1 


\section{Freie Beiträge}

\section{zur Jahrestagung der Gesellschaft für Musikforschung 2019}

Herausgegeben von Nina Jaeschke und Rebecca Grotjahn

Detmold: Musikwissenschaftliches Seminar der Universität Paderborn und der Hochschule für Musik Detmold 2020 
DOI: $10.25366 / 2020.42$

Online-Version verfügbar unter der Lizenz: Urheberrecht 1.0, $<$ https://rightsstatements.org/page/InC/1.0/?language=de>

Bibliografische Information der Deutschen Nationalbibliothek

Die Deutsche Nationalbibliothek verzeichnet diese Publikation in der Deutschen Nationalbibliografie; detaillierte bibliografische Daten sind im Internet über http://dnb.d-nb.de abrufbar.

\section{Impressum}

Redaktion: Nina Jaeschke, Rebecca Grotjahn und Jonas Spieker Satz: Nina Jaeschke

(C) Musikwissenschaftliches Seminar der Universität Paderborn und der Hochschule für Musik Detmold 2020 


\section{INHALT}

Vorwort $\quad$ IX

Komponieren für das Radio: Akteure, Diskurse, Praktiken $\quad 1$

Musikwissenschaft - Feminismus - Kritik: Ein Generationenaustausch 6

\section{Stefan Alschner}

Der Wagner-Sänger Joseph Aloys Tichatschek - Vom Nachlass zum Netzwerk

\section{Alenka Barber-Kersovan}

Songs for the Goddess. Das popmusikalische Neo-Matriarchat zwischen Ethno-Beat,

erfundenen Traditionen und kommerzieller Vermarktung

Elias Berner, Julia Jaklin, Peter Provaznik, Matej Santi, Cornelia Szabó-Knotik

Musikgeschichte anders erzählen? Das Beispiel der 1970er in Österreich.

Musikhistoriographie in der Zeit der Digitalisierung

\section{Mauro Fosco Bertola}

„Ein Laut so klagevoll”. Lohengrin zwischen Richard Wagner und Salvatore Sciarrino

\section{Matthieu Cailliez}

Europäische Rezeption der Berliner Hofoper und Hofkapelle von 1842 bis 1849

\section{lacopo Cividini}

Zwischen klassischer Musikphilologie und angewandter Informatik:

Die Digitale Mozart-Edition (DME) der Stiftung Mozarteum Salzburg

\section{Marko Deisinger}

Fortschrittliche Technologie im Dienste eines Antimodernisten.

Heinrich Schenker und der österreichische Rundfunk

\section{Norbert Dubowy}

Vom Kritischen Bericht zur Kritischen Dokumentation am Beispiel der Digital-interaktiven Mozart-Edition

\section{Markus Engelhardt}

Musik zwischen Nation Building und Internationalität. Italien um 1900

\section{Maryam Haiawi}

Das Oratorium im Spannungsfeld der Konfessionen: 


\section{Judith I. Haug}

"Manch eine*r liegt, morgens noch trunken, im Rosengarten" - Rekonstruktionen

osmanischer Musikgeschichte in Gesangstextsammlungen

\section{Renate Koch}

Marcel Prawy und das erste Broadway-Musical im Österreich der Nachkriegszeit

Susanne Kogler, Julia Mair, Juliane Oberegger, Johanna Trummer

Erich Marckhl - Musikausbildung in der Steiermark nach 1945.

Brüche und Kontinuitäten

\section{Marie-Anne Kohl}

Die weinende Jury. "Geschlechtslose" Tränen bei globalen Musik-Castingshows?

\section{Fabian Kolb}

Tanztheater und filmische Ästhetik. Cineastische Einflüsse und Gestaltungsweisen in den Kompositionen für die Ballets Suédois 1920-1925

\section{Christian Lehmann}

Tempobezeichnungen von Julius Stockhausen für Die schöne Müllerin:

Ein Quellenfund

\section{Martin Link}

Signum et gens - Zur Gendersemiotik in Clara und Robert Schumanns Liederzyklus Liebesfrühling

\section{Livio Marcaletti}

„Strafspiel" und satirische Stilmittel in musikdramatischen Gattungen des frühen 18. Jahrhunderts

\section{Tobias Marx, Martin Lissner}

Thüringer Musikszene - Jugendmusikredaktionen als außerschulische musikbezogene Bildungskontexte

\section{Maho Naito}

Die Parallelität der Entstehungsprozesse der ersten beiden Symphonien Gustav

Mahlers: Instrumentation, Revision und Dirigierpraxis

\section{Elisa Novara}

Eine Schumann-Werkstatt? Zur Übertragbarkeit der Methoden vom Projekt 
Theodora Oancea, Joachim Pollmann, Jonas Spieker

Kollaborateure - Involvierte - Profiteure. Erarbeitung eines Online-Lexikons zur

Musik in der NS-Zeit

\section{Kiron Patka}

„Ich wollte eigentlich Sängerin werden." Berufsselbstbilder von Tontechniker*innen im Radio

\section{Siegwart Reichwald}

Die Leiden der jungen Clara: Das Klaviertrio Opus 17 als Ausdruck einer Neu-

Romantikerin

\section{Elisa Ringendahl}

Lied versus Oper - Pole musikalischer Gattungen bei Oscar Bie

\section{Benedikt Schubert}

Struktur und Exegese. Über Eigentümlichkeiten in der Arie "Des Vaters Stimme ließ sich hören" (BWV 7/4)

Uwe Seifert, Sebastian Klaßmann, Timo Varelmann, Nils Dahmen

Computational Thinking in der Musikwissenschaft: Jupyter Notebook als Umgebung

für Lehre und Forschung

\section{Yusuke Takamatsu}

Synthese als Modus der Prozessualität bei Schubert:

Sein spezifisches Wiederholungsprinzip im langsamen Satz

\section{Daniel Tiemeyer}

Johann Nepomuk Hummels Sonate in fis-Moll Op. 81 - Studien zu Entstehungs-

hintergrund, Rezeption und formalerStruktur

\section{Andrea van der Smissen}

Musikalische Innovation im Umfeld der Moderne und historischen Avantgarde in Ungarn

\section{Tim Ziemer, Holger Schultheis}

Psychoakustische Sonifikation zur Navigation in bildgeführter Chirurgie

\section{Magdalena Zorn}

Musik mit dem Radio hören: Über den Begriff der musikalischen Aufführung 
Gabriele Buschmeier in memoriam 


\section{Vorwort}

Die vorliegenden Bände dokumentieren die Jahrestagung der Gesellschaft für Musikforschung 2019. In den dreieinhalb Tagen vom 23. bis zum 26. September 2019 wurden in Paderborn und Detmold nicht weniger als 185 Beiträge präsentiert, verteilt auf diverse Symposien, Round tables, Freie Sektionen und Postersessions. Sie alle auf einen Nenner bringen zu wollen, ist ein Ding der Unmöglichkeit - und das ist gut so, ist es doch Ziel der Jahrestagungen, die große Vielfalt der Themen und Methoden des Faches Musikwissenschaft abzubilden. Um die thematische Vielfalt der freien Referate angemessen abbilden zu können und gleichzeitig den inhaltlichen Schwerpunkten der beiden hier publizierten Hauptsymposien ausreichend Raum bieten zu können, erscheinen diese in drei Bänden.

„Musikwissenschaft: Aktuelle Perspektiven": Der Titel der kleinen Reihe ist keine Verlegenheitslösung. Musikwissenschaft im Kontext der Digital Humanities; Musikwissenschaft und Feminismus; Musik und Medien; Musikalische Interpretation - schon die Felder, die von den vier Hauptsymposien bespielt wurden, wären noch vor wenigen Jahrzehnten allenfalls an der Peripherie das Faches zu finden gewesen. Sie entsprechen Arbeitsschwerpunkten der Lehrenden am Musikwissenschaftlichen Seminar der Universität Paderborn und der Hochschule für Musik Detmold, das die Tagung ausrichtete. Zugleich nehmen sie Bezug auf aktuelle Ereignisse und Entwicklungen. So erwuchs das von Andreas Münzmay und Joachim Veit organisierte Symposium „Brückenschläge - Informatik und Musikwissenschaft im Dialog" unmittelbar aus den Erfahrungen im Virtuellen Forschungsverbund Edirom (ViFE) und im fakultäten- und hochschulübergreifenden Zentrum Musik-Edition-Medien (ZenMEM). Der 200. Geburtstag von Clara Wieck/Schumann war der Anlass für das von Rebecca Grotjahn geleitete Symposium „Die Begleiterin - Clara Schumann, Lied und Liedinterpretation", das in enger Kooperation mit der Hochschule für Musik Detmold durchgeführt wurde. Das Hauptsymposium „Brückenschläge" wird in einem separaten Band publiziert (Bd. 3 der vorliegenden Reihe). Im Rahmen dieses Symposiums führte die von Stefanie Acquavella-Rauch geleitete Fachgruppe Digitale Musikwissenschaft eine Posterpräsentation durch, die von den Beiträger*innen erfreulicherweise zu kürzeren Texten umgearbeitet wurden, sodass sie hier ebenfalls, zusammen mit den Postern,

publiziert werden können. Hinzu kommen einige Beiträge, die bereits bei der Jahrestagung 2018 in Osnabrück präsentiert wurden. Auch das Hauptsymposium "Die Begleiterin" wird in einem eigenen Band (Bd. 2) publiziert. Die Beiträge zu den beiden anderen Hauptsymposien hingegen werden an anderen Orten veröffentlicht; in Band 1 („Freie Beiträge zur Jahrestagung der Gesellschaft für Musikforschung 2019") der vorliegenden Publikation finden sich jedoch Einführungen und Abstracts. Das Symposium „Komponieren für das Radio" unter Leitung von Antje Tumat und Camilla Bork (Katholieke Universiteit Leuven) behandelte Einflüsse des Mediums auf Kompositionsprozesse sowie durch radiophone Kompositionen bzw. radiophonen Klang ausgelöste Diskurse. Sarah Schauberger und Cornelia Bartsch (Universität Oldenburg) nahmen das 25-jährige Jubiläum der Fachgruppe Frauen- und Genderstudien zum Anlass für einen Generationenaustausch zum Thema "Musikwissenschaft - Feminismus - Kritik": Was wa- 
ren vor einem Vierteljahrhundert Inhalte und Aufgaben einer feministischen Musikwissenschaft und wie kann sich diese heute positionieren?

Bewusst haben wir im Tagungsbericht auf inhaltliche Eingriffe in die Beiträge verzichtet. ${ }^{1}$ Das gilt besonders für die Freien Referate: Es galt, den Charakter der Jahrestagung als Forum für ,freie', d. h. innovative und auch experimentelle Gedanken zu wahren. Einige Kolleg*innen, die die Tagung mit Vorträgen und Posterpräsentationen bereichert hatten, haben sich gegen eine Publikation im vorliegenden Band entschieden - sei es, weil sie eine Möglichkeit fanden, ihre Beiträge in einem inhaltlich passenderen Rahmen zu veröffentlichen, sei es, weil ihre Überlegungen in ihre entstehenden Qualifikationsschriften fließen sollen, oder sei es, weil sie von den Autor*innen in der vorgetragenen Form zunächst verworfen wurden. Auch damit erfüllt eine Freie-Referate-Sektion ihren Zweck: Die Diskussionen mit der versammelten Fach-Öffentlichkeit sollen dabei helfen, Gedanken weiterzuentwickeln und zu verändern. In diesem Sinne sei allen Beteiligten - den Autor*innen, den nichtpublizierenden Referent*innen und den Mit-Diskutant*innen - ganz herzlich gedankt für ihr Mitwirken bei der Tagung.

Unser herzlicher Dank gilt einer Reihe weiterer Personen, die zum Gelingen dieser drei Bände beigetragen haben. Hier ist besonders Jonas Spieker zu nennen, der uns tatkräftig bei der Redaktion geholfen hat. Andrea Hammes (SLUB Dresden) sei herzlich für die Aufnahme unseres Bandes auf musiconn.publish gedankt - wir freuen uns, damit unsererseits zur Etablierung dieser innovativen Publikationsplattform beizutragen.

Erneut möchten wir an dieser Stelle allen Menschen danken, die uns bei der Organisation, Ausrichtung und Finanzierung der Tagung selbst unterstützt haben: der Präsidentin der Universität Paderborn, Prof. Dr. Birgitt Riegraf, dem Rektor der Hochschule für Musik Detmold, Prof. Dr. Thomas Grosse, den Kolleginnen und Kollegen der beiden beteiligten Hochschulen, dem Vorstand der Gesellschaft für Musikforschung, der Universitätsgesellschaft Paderborn und allen Sponsoren. Besonders dankbar sind wir den Mitarbeiter*innen und den studentischen bzw. wissenschaftlichen Hilfskräften des Musikwissenschaftlichen Seminars, die bei der Vorbereitung und Ausrichtung der Tagung immensen Einsatz zeigten - stellvertretend sei an dieser Stelle Johanna Imm erwähnt, die zusammen mit Nina Jaeschke das Herz des Organisationsteams bildete.

Wir widmen diese Reihe Dr. Gabriele Buschmeier, dem langjährigen Vorstandsmitglied der Gesellschaft für Musikforschung, die kurz vor der Publikation dieses Bandes unerwartet verstarb.

Detmold, im September 2020

Rebecca Grotjahn und Nina Jaeschke

Zitation: Rebecca Grotjahn und Nina Jaeschke, „Vorwort”, in: Freie Beiträge zur Jahrestagung der Gesellschaft für Musikforschung 2019, hrsg. von Nina Jaeschke und Rebecca Grotjahn (= Musikwissenschaft: Aktuelle Perspektiven. Bericht über die Jahrestagung der Gesellschaft für Musikforschung 2019 in Paderborn und Detmold, Bd. 1), Detmold 2020, S. IX-X, DOI: 10.25366/2020.43.

1 Freigestellt war den Autor*innen auch, ob sie sich für eine gendersensible Sprache entscheiden bzw. welche Form des Genderns sie bevorzugen. 